\title{
Harvesting the wisdom of crowds for election predictions using the Bayesian Truth Serum
}

\author{
Henrik Olsson ${ }^{1, a}$, Wändi Bruine de Bruin ${ }^{\mathrm{b}}$, Mirta Galesic $^{\mathrm{a}}$, \& Drazen Prelec ${ }^{\mathrm{c}}$

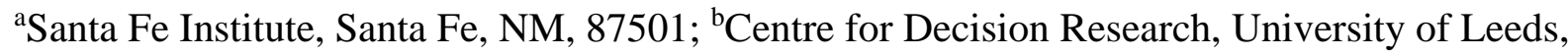 \\ Leeds LS2 9JT, UK; and 'Sloan School of Management, Massachusetts Institute of Technology, \\ Cambridge, MA 02142.
}

\begin{abstract}
Social scientists, journalists, politicians, policy makers, businesses, and the general public rely on surveys to understand changing attitudes and behaviors in the society. Prominent among these are election polls. Most election polls ask people for which candidate they will vote (own intention questions). Accuracy of election predictions can be further improved by leveraging the wisdom of crowds: asking people whom they expect will win (election-winner expectations) or whom their friends will vote for (social-circle expectations). Here we investigate a complementary approach that uses the Bayesian Truth Serum (BTS) scoring algorithm and election-winner expectations to improve survey predictions based on own intentions and socialcircle expectations. We present a new theorem that gives conditions under which moderate weighting of respondents by their BTS scores should improve survey accuracy even if everyone responds honestly. In a national longitudinal survey $(\mathrm{N}>4,000)$ conducted in three waves before the 2018 US House of Representatives election, we compared predictions based on own intentions, state-winner and social-circle expectations, and BTS-scored expectations. Socialcircle expectations outperformed own intentions at the national and state levels in predicting the vote-share distribution and the margin between Democrats and Republicans. BTS scores provided additional improvements to predictions of national and state-level vote-share distributions based on social-circle expectations.
\end{abstract}

${ }^{1}$ Corresponding author: Henrik Olsson, Santa Fe Institute, 1399 Hyde Park Rd., Santa Fe, NM 87501. Email: olsson@santafe.edu 


\section{Introduction}

Surveys of behaviors and opinions have an important societal role. They inform citizens about their place in our changing society, scientists about possible mechanisms underlying individual and social behaviors, journalists about the topics people care about, and policy and business professionals about public needs and potential for acceptance of different initiatives and products. Prominent examples include surveys of health $(1,2)$, economic outlook and consumer sentiment (3), and values (4). Here we focus on election polls which are of broad general interest because they inform voters about viable candidates, influence their campaign contributions and tactical voting decisions, enable politicians to assess support for different positions and design their campaigns, and allow households and business to plan for the future while accounting for possible actions of a next government (5).

Traditional election polls ask people about their own voting intentions. Although the accuracy of election polls has not declined appreciably (6), recent notable prediction failures have highlighted the limitations of traditional methods. For example, polling errors in several key states contributed to the general prediction of a Clinton victory in the 2016 U.S. presidential election (7). Several explanations have been proposed for these errors, including substantive changes in voting preferences during final days before the election, inadequate adjustment for the overrepresentation of specific population segments, and reluctance among some voters to reveal their voting preferences before the election (7). Because these issues might be difficult to overcome with traditional polling, it is important to explore new methods.

Wisdom-of-crowds methods can be used to improve election predictions. One such method treats respondents as 'citizen forecasters' and asks them to predict the election outcome (8-10). In principle, these election-winner expectation questions allow respondents to go beyond their own voting preferences and summarize all potentially relevant information that they possess about the elections (11-15). However, election-winner expectations do not always improve polling accuracy (16). The method is vulnerable to an echo-chamber effect if respondents merely repeat the predictions they hear in the media (17).

A related, but more selective approach is to ask respondents to act as 'informants' and predict the voting of only their social circle, including family, friends, and acquaintances (16). Recently, we have found that the average of these social-circle expectations from national probabilistic samples was more accurate than the predictions based on own intention questions 
for the 2016 U.S. Presidential Election, the 2017 French Presidential Election (16), the 2017 Dutch general election, and the 2018 Swedish general election (18). Furthermore, social-circle expectations improved predictions of state election results and predicted individual voting behavior beyond own intentions and election-winner expectations. Social-circle expectation questions rely on respondents' direct experiences with their immediate social environments (8, 19, 20). They provide information about individuals within respondents' social circles who might otherwise not be included in surveys, and they anticipate changes in own voting intentions that could occur over time because of processes of social influence $(15,21-24)$. This additional information provided by social-circle expectations might be especially important with small samples (6).

An issue of concern with all wisdom-of-crowds methods is how to take into account variation in respondents' competence. It is well known that simple averaging of answers is biasing when some answers reflect better information than others (25). To address this problem, methods have been developed that encourage careful and attentive responding (26), identify expert respondents $(27)$, and incentivize honest responding $(28,29)$. These methods have not yet been applied to election polls, even though it is likely that surveyed respondents differ in their awareness of candidates and positions, knowledge of social circles, and general conscientiousness in answering questions.

Here we test whether the Bayesian Truth Serum (BTS) (30), a scoring method developed for incentivizing honest answers to any type of non-verifiable question (e.g., 9-13), can also improve the accuracy of election polls by applying weights to the respondents' answers. BTS scores have already been successfully implemented with product adoption surveys (36). A related BTS-inspired filtering model has been tested in market research (37), and is discussed in more detail below. BTS scoring is ex-ante incentive-compatible, which means that honest answers maximize respondents' expected scores $(31,38)$ even though the survey administrator has no direct information about honesty. However, BTS scores are not ex-post neutral, which means that even if all respondents answer honestly, some will receive higher scores by chance. For this reason, the theoretical results in (30) alone do not justify weighting or filtering respondents by their BTS scores. Some additional theory is needed.

We begin by defining an application of BTS designed to enhance detection of deceptive vs. honest responding. We then derive a new theorem that gives conditions under which 
moderate weighting of respondents' answers by their BTS scores should improve prediction accuracy even if all respondents answer honestly. Our application pertains to data from the Understanding America Study (UAS) (39), conducted in three waves before, and one wave after the 2018 United States House of Representatives election. By comparing intentions to vote in the first three waves with self-reported voting behavior after the election, we investigate two research questions. First, we test whether BTS scores can identify respondents that are wellcalibrated, that is who can accurately predict their own actual voting behavior. Second, we test whether BTS-scored own intentions and social-circle expectations yield more accurate election forecasts at the state and national levels than own intentions, social-circle expectations, and statewinner expectations.

Further results, including on how different methods depend on sample size and survey timing, preregistered hypotheses about the success of different methods (40), and results regarding individual voting behavior that replicate the results in (16) are presented in the SI.

\section{Theoretical results: Extending the Bayesian Truth Serum (BTS)}

In its original setting (30), BTS scoring applies to a single multiple-choice item with $m$ possible options. Each respondent answers two questions. The first question asks respondents for an endorsement of one option on the multiple-choice question. In the case of election polls, this can be a question about the candidates or parties they or their friends will vote for. The second question asks for respondents' prediction of distribution of answers given by others. In the context of election polls, this is their expectation of how other people in the survey or in their state will vote.

The underlying theory assumes that honest answers to the primary question drive honest answers to the secondary, prediction question. That is, people with the same primary answer should make (roughly) the same predictions of others' answers. The theory further assumes that respondents' predictions are computed by Bayesian updating from a common prior, treating their own primary answer as a 'sample-of-one.' Their predictions of others' answers are the expectations of this Bayesian posterior distribution.

The score, $u^{r}$, of the own intention answer $x_{k}^{r}$ for participant $r$ is given by:

$$
u^{r}=\sum_{k=1}^{m} x_{k}^{r} \log \frac{\bar{x}_{k}}{\bar{y}_{k}}+\sum_{k=1}^{m} \bar{x}_{k} \log \frac{y_{k}^{r}}{\bar{x}_{k}}+\lambda \sum_{k=1}^{m} \bar{y}_{k}^{r} \log \frac{y_{k}^{r}}{\bar{y}_{k}^{r}}
$$


where $x_{k}^{r}$ is the answer to the own intention question, with value 1 if respondent $r$ has declared an intention to vote for $k=$ Republican, Democrat, or Other, and value zero otherwise. $\bar{x}_{k}$ is the arithmetic mean of own intentions across all respondents in a given state, $\bar{y}_{k}$ is the geometric mean of the answers to the state-winner expectation question in a given state, $y_{k}^{r}$ is the statewinner expectation for respondent $r$ and question $k$, and $\bar{y}_{k}^{r}$ the geometric mean prediction of respondents selecting the same answer, excluding respondent $r$. Scores for the social-circle expectations are calculated in the same way, but with own intentions substituted by social-circle expectations (see Materials and Methods for additional details).

The first part of Eq. 1 gives the information score for the reported own intention. An honest answer maximizes a participant's expected information score if other participants are answering honestly and the sample is large (30). The second part gives the prediction score for the respondents' expectations of others' answers. Here, we approximate these expectations by respondents' state-winner expectations. Together, the information score and prediction score give the BTS score.

The last part of Eq. 1 extends (30) with a divergence penalty, a term taken from the Divergence-based Bayesian Truth Serum (34). We will therefore refer to the total score calculated in Eq. 1 as the BTSD score (see also 33). The divergence term compares a respondent's predictions with predictions made by those who reported the same intentions, and penalizes any discrepancies. Under the assumptions of the model, respondents with the same intentions will provide the same predictions and the divergence penalty will be zero. Therefore, BTS and BTSD generate identical scores if everyone answers honestly and the assumptions of the model hold.

Next we derive a new result that supports BTS weighting even if all respondents answer honestly. Following (37), we assume that respondents may be divided into two categories, the relatively more informed and relatively less informed. In (37) the more informed respondents were presumed to know the distribution of honest answers perfectly, and could therefore be identified by their superior prediction scores. Here we assume that the less informed respondents are sampled from the prior distribution while the more informed respondents are sampled from the 'actual' distribution, i.e., one that matches the eventual election result. With any type of question the less informed category may include respondents who are simply careless or unmotivated to answer. 
The best election forecast would be obtained by sampling only the more informed respondents, if one could identify them ahead of time. Even though this is not possible, one can improve on an unweighted election forecast by giving more weight to respondents with high BTS scores. The appropriate weighting formula and theorem are stated below.

Proposition Let $\bar{x}_{k}(\beta)$ denote the exponentially BTS-weighted average of answers:

$$
\bar{x}_{k}(\beta)=\frac{\sum_{r} \exp \left(\beta u^{r}\right) x_{k}^{r}}{\sum_{j=1}^{m} \sum_{r} \exp \left(\beta u^{r}\right) x_{j}^{r}}
$$

If a fraction $\varepsilon>0$ of respondents are sampled from the prior, while the remaining $(1-\varepsilon)$ are sampled from the actual distribution, then for all $\beta$ in an interval: $0<\beta<\beta^{\circ}, \bar{x}_{k}(\beta)$ will be closer to the actual distribution than the unweighted average $\bar{x}_{k}(\beta=0)$.

'Closer' means that for any pair of candidates, $k$ and $j$, the ratio $\bar{x}_{k}(\beta) / \bar{x}_{j}(\beta)$ is closer to the truth than the unweighted ratio (proof in Appendix). Under model assumptions, informed respondents will always have higher scores on average than uninformed respondents. Because informed respondents represent the actual distribution, giving them more weight improves survey accuracy.

Consider an example with two candidates, A and B, a uniform prior and an actual distribution 60-40 in favor of $\mathrm{A}$. In that case, if $\varepsilon=.5$, the survey results will yield a prediction of 55-45 (as the less informed respondents are sampled from the prior and divide 50-50 between A and B). The BTS score (Eq. 1) will reward those whose frequency is higher relative to the prior, that is, respondents expressing intentions to vote for A. This will move the weighted average $\bar{x}_{k}(\beta)$ from 55-45 towards the true 60-40 split. However, if $\beta$ is too high then the weighted average will overshoot the actual 60-40. The Proposition asserts that the directional benefit of BTS weighting holds for any number of voting options and any prior distribution.

Reasonable $\beta$ values can be derived from assumptions about the prior and fraction of less informed respondents. An optimal $\beta$ minimizes average root-mean-squared error (RMSE) of estimated-versus-actual vote percentages, with average taken according to the hypothesized prior distribution. For example, with a uniform prior, $\beta=0.17$ is optimal for $\varepsilon=0.1$, and $\beta=0.38$ for $\varepsilon=0.2$. A tighter prior, e.g., a beta-distribution with mean 0.40 and standard deviation 0.06 , yields lower optimal $\beta$ values, in this case $\beta=0.11$ for $\varepsilon=0.1$, and $\beta=0.25$ for $\varepsilon=0.2$. 


\section{Empirical results: Predicting the 2018 US election}

\section{Do BTS scores reflect how well respondents' own intentions are calibrated with their actual voting behavior?}

Our results suggest that BTS and BTSD scores measure credibility of probabilistically stated intentions to vote, in the sense of improving calibration, and that weighting by these scores could improve overall poll accuracy. We investigated how calibrated participants are by comparing their own intentions stated before the elections with their actual voting behavior reported after the elections. Perfect calibration implies that own intention of $x \%$ chance of an action (voting for a particular candidate or not voting at all) translates into an $x \%$ chance of actually performing that action at the time of elections.

Figure 1 shows the calibration curves for BTSD (BTS curves show the same pattern of results). The BTSD score distribution is then split into five quantiles, and the frequency of actions plotted separately for each quantile on the $y$-axis. It is evident that participants in the lowest BTSD score quintile is the most miscalibrated, as measured by the deviations from the identity line. For example, own intentions of $100 \%$ probability of performing an action correspond to an $80 \%$ chance of actually performing that action. The top three BTSD score quintiles look almost identical, and suggest slight underconfidence (i.e., they have an S-shaped curve). For these respondents a stated probability of $70 \%$ correspond to a $90 \%$ chance of actually performing the action. In addition, BTS scores interact with stated probabilistic intentions in a logistic regression predicting respondents actual voting behavior $(\mathrm{p}<.001)$ 

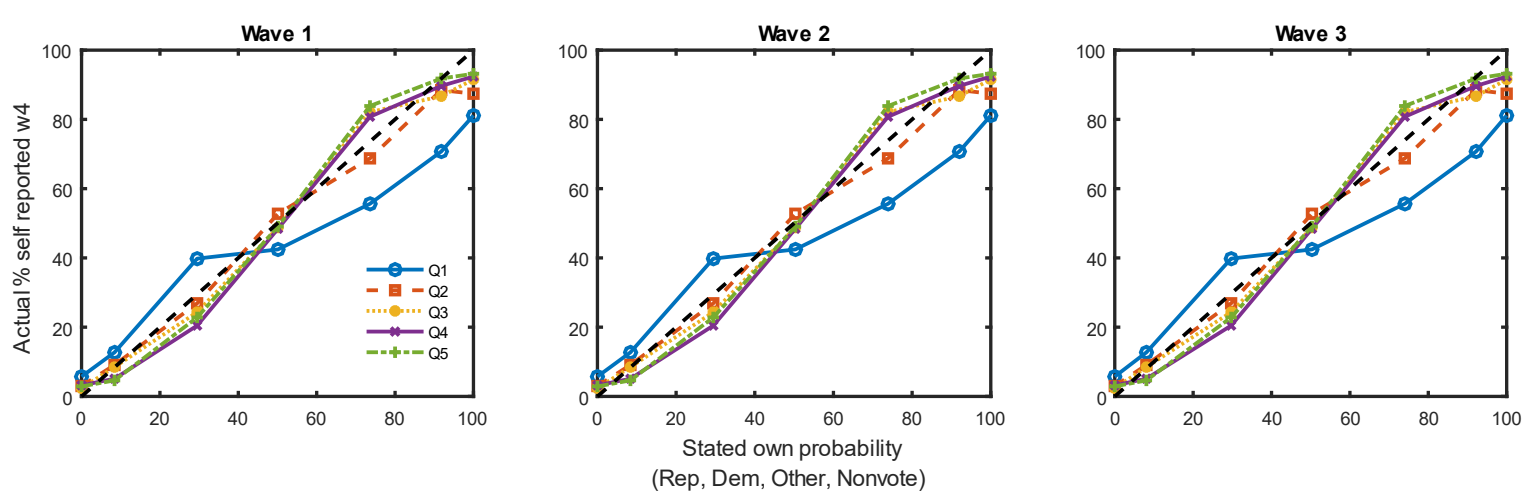

Figure 1. Calibration curves for the predictions of BTSD for self-reported voting behavior (BTS curves show the same pattern). The $x$-axis divides probabilities respondents assign to their own future action (to vote for a particular party, or not vote at all) into seven discrete bins (with probability intervals: $0,1-20,21-40,41-60,61-80,81-99$, and 100\%). The $y$-axis shows the frequency (percent) of times actions in a given bin are actually reported in the post-election survey. Perfect calibration is defined by the $45^{\circ}$ dashed identity line. Deviations from that line indicate miscalibration of own stated intentions. The data for those who voted Republican, Democrat, Other, and Nonvoters are collapsed together.

\section{Are BTS-scored own intentions and social-circle expectations more accurate than own intentions, social-circle expectations, and state-winner expectations?}

We compared different predictions of 2018 election results on both national and state levels. National predictions were obtained as average of state-level predictions, weighted by the size of a state population 18+ (Table 1), as well as based on the whole survey sample (Table S1; see Materials and Methods for details). Essentially, the national level is an analysis with $N=1$ and the state level is an analysis with $N=50$. We calculated two types of errors, both frequently used in polling studies $(41,42)$. One is the average absolute error of predictions of vote shares for all parties (also called Mosteller 3): Democrat, Republican, and Other. The other is the absolute error of the Democratic-Republican (D-R) margin (also called Mosteller 5 (41)). Each type of error was calculated for national-level predictions (Figure 2 and Table S2) and for state-level predictions (Figure 3 and Table S3).

Own intentions. On the national level, BTS applied to own intentions improved the accuracy of predictions for all parties (Figure 2A). However, it did worse than own intentions for the D-R margin (Figure 2B). Across all waves, BTS-scored own intentions did better than state- 
winner expectations for all parties, and better than state-winner expectations for the D-R margin for waves 2 and 3.

BTSD applied to own intentions improved the accuracy compared to BTS for national predictions for all parties (Figure 2A). However, BTSD did worse than BTS for the national prediction of the D-R margin (Figure 2B). Across all waves, BTSD did better than state-winner expectations for all parties, and better than state-expectations for the D-R margin for waves 2 and 3.

On the state level, BTS applied to own intentions did not improve accuracy for all parties in any of the waves (Figure 3A), with confidence intervals that included zero for wave 1 and wave 3 (Figure S1 column 2). It also performed worse than own intentions for the D-R margin (Figure 3B), with confidence intervals that did not include zero (Figure S2 column 2). BTS also performed worse than state-winner expectations for all waves both for all parties and the D-R margin.

BTSD applied to own intentions had similar performance as own intentions for all parties on the state level (Figure 3A), with confidence intervals that included zero for Wave 1 and Wave 3 (Figure S1 column 4). BTSD did worse than own intentions for the D-R margin (Figure 3B), with confidence intervals that did not include zero (Figure S2 column 4). BTSD also performed worse than state-winner expectations for all waves both for all parties and the D-R margin.

Social-circle expectations. On the national level, BTS applied to social-circle expectations improved the accuracy of social-circle expectations for all parties (Figure 2A). However, it did worse than social-circle expectations for the D-R margin for Wave 1 and 2 (Figure 2B). Across all waves, BTS did better than state-winner expectations for all parties and for the D-R margin.

BTSD applied to social-circle expectations improved the accuracy compared to BTS for all parties on the national level (Figure 2A). In fact, BTSD-scored social-circle expectations outperformed all other methods for all parties. For the D-R margin, BTSD only outperformed BTS for wave 1 (Figure 2B). Across all waves, BTSD did better than state-winner expectations for all parties and for the D-R margin.

On the state level, BTS applied to social-circle expectations improved the accuracy of social-circle expectations for all parties (Figure 3A), but confidence intervals for all waves included zero (Figure S1 column 3). For the D-R margin BTS was less accurate than social-circle 
expectations for Wave 1 and had similar levels of accuracy for Wave 2 and 3 (Figure 3B), with all confidence intervals including zero. BTS performed better than state-winner expectations for all parties for wave 1 and wave 3. For the D-R margin, state-winner expectations performed better than BTS for all waves.

BTSD applied to social-circle expectations improved the accuracy of social-circle expectations for all parties, but again the confidence intervals for all waves included zero (Figure S1 column 5). For the D-R margin, BTSD was less accurate than social-circle expectations for Wave 1 and Wave 3 and more accurate for wave 1 (Figure 3B), again with all confidence intervals including zero (Figure S2 column 5). BTSD performed better than state-winner expectations for all parties for wave 1 and wave 3 and similar for wave 2. For the D-R margin, state-winner expectations performed better than BTS for all waves.

\section{Auxiliary Analyses}

Sample size effects on the accuracy of predictions. Figures S3 and S4 show the relationship between error for different states and the survey sample size in each state. Socialcircle expectations, BTS and BTSD applied to social-circle expectations, and state-winner expectations are the ones that are least affected by sample size. Own intentions and BTS and BTSD applied to own intentions are the worst performing when sample size is small.

Survey timing. For predicting national vote shares for all parties, the advantage of social-circle expectations and their BTS variants over own intentions was larger in earlier than in later survey waves (Figure 2A). However, for the D-R margin on the national level (Figure 2B), there was no clear pattern of a social-circle advantage in earlier waves.

For predicting state results, there were no advantages of social-circle expectations and their BTS variants over own intentions in predicting either voter shares of all parties or the D-R margin in earlier than in later survey waves (Figure 3).

Preregistered hypotheses. Our preregistered hypotheses were mostly confirmed, with some exceptions (see SI). Predictions based on social-circle expectations were overall more accurate than predictions based on own intentions (H1a). Contrary to our hypothesis, statewinner expectations were mostly worse than own intentions for predictions on the national level (H1b). In line with our hypothesis, state-winner expectations consistently outperformed own intentions on the state level both for all parties and the D-R margin. Contrary to our hypothesis, BTS-scored own intentions did not improve predictions for most of the comparisons (H2a). BTS- 
scored social circle expectations, however, improved the predictions for most comparisons (H2b). Social circle expectations predicted individual voting behavior over and above own intentions and state-winner expectations (H3). These results on individual voting behavior (see regression tables in SI) replicate those in (16). 
Table 1

National predictions, calculated as weighted average of state predictions

\begin{tabular}{|c|c|c|c|c|c|c|c|c|}
\hline & $\begin{array}{r}\text { Election } \\
\text { results (Nov } \\
6 \text { ) results } \\
\end{array}$ & $\begin{array}{r}\text { Own } \\
\text { intentions } \\
\end{array}$ & $\begin{array}{l}\text { Social-circle } \\
\text { expectations }\end{array}$ & $\begin{array}{l}\text { State-winner } \\
\text { expectations }\end{array}$ & $\begin{array}{l}\text { BTS own } \\
\text { intentions }\end{array}$ & $\begin{array}{r}\text { BTS social- } \\
\text { circle } \\
\text { expectations }\end{array}$ & $\begin{array}{r}\text { BTSD own } \\
\text { intentions }\end{array}$ & $\begin{array}{r}\text { BTSD } \\
\text { social-circle } \\
\text { expectations } \\
\end{array}$ \\
\hline \multicolumn{9}{|c|}{$\begin{array}{l}\text { Wave 1 (Aug 22 - } \\
\text { Sep } 11, N=4,511)\end{array}$} \\
\hline Republicans & 44.8 & 39.2 & 42.2 & 42.5 & 39.0 & 42.5 & 39.1 & $\underline{42.6}$ \\
\hline Democrats & 53.4 & 51.8 & 49.7 & 47.4 & 52.3 & 49.9 & 52.6 & $\underline{50.0}$ \\
\hline $\begin{array}{l}\text { Other } \\
\text { Democrats- }\end{array}$ & 1.7 & 9.0 & 8.1 & 10.1 & 8.7 & 7.6 & 8.3 & $\underline{7.3}$ \\
\hline $\begin{array}{l}\text { Republicans } \\
\text { Wave } 2 \text { (Sep } \\
\text { Oct } 4, N=4,25\end{array}$ & 8.6 & 12.6 & $\underline{7.5}$ & 4.9 & 13.2 & 7.3 & 13.6 & 7.4 \\
\hline Republicans & 44.8 & 40.5 & 42.5 & 43.1 & 40.5 & 42.8 & 40.6 & $\underline{42.8}$ \\
\hline Democrats & 53.4 & 51.8 & 50.4 & 48.4 & 51.9 & 50.4 & 52.1 & $\overline{50.4}$ \\
\hline $\begin{array}{l}\text { Other } \\
\text { Democrats- }\end{array}$ & 1.7 & 7.7 & 7.1 & 8.5 & 7.6 & 6.9 & 7.3 & $\underline{6.7}$ \\
\hline $\begin{array}{l}\text { Republicans } \\
\text { Wave } 3 \text { (Oct } \\
\text { Nov } 5, N=5,0\end{array}$ & 8.6 & 11.3 & $\underline{7.8}$ & 5.2 & 11.5 & 7.6 & 11.6 & 7.6 \\
\hline Republicans & 44.8 & 41.1 & 42.2 & 43.1 & 41.2 & 42.5 & $\underline{41.3}$ & 42.6 \\
\hline Democrats & 53.4 & 53.2 & 51.2 & 47.9 & 53.4 & 51.2 & $\underline{53.5}$ & 51.2 \\
\hline $\begin{array}{l}\text { Other } \\
\text { Democrats- }\end{array}$ & 1.7 & 5.7 & 6.6 & 9.0 & 5.4 & 6.3 & $\underline{5.2}$ & 6.1 \\
\hline Republicans & 8.6 & 12.2 & 9.1 & 4.8 & 12.3 & 8.6 & 12.2 & $\underline{8.6}$ \\
\hline
\end{tabular}

Note. BTS = Bayesian Truth Serum. BTSD = Bayesian Truth Serum with divergence score (see Eq. 1). Bold underline indicates predictions with lowest error within the USC survey (see Figure 1 for the errors). National predictions are average of state predictions weighted by the size of a state population $18+$. 

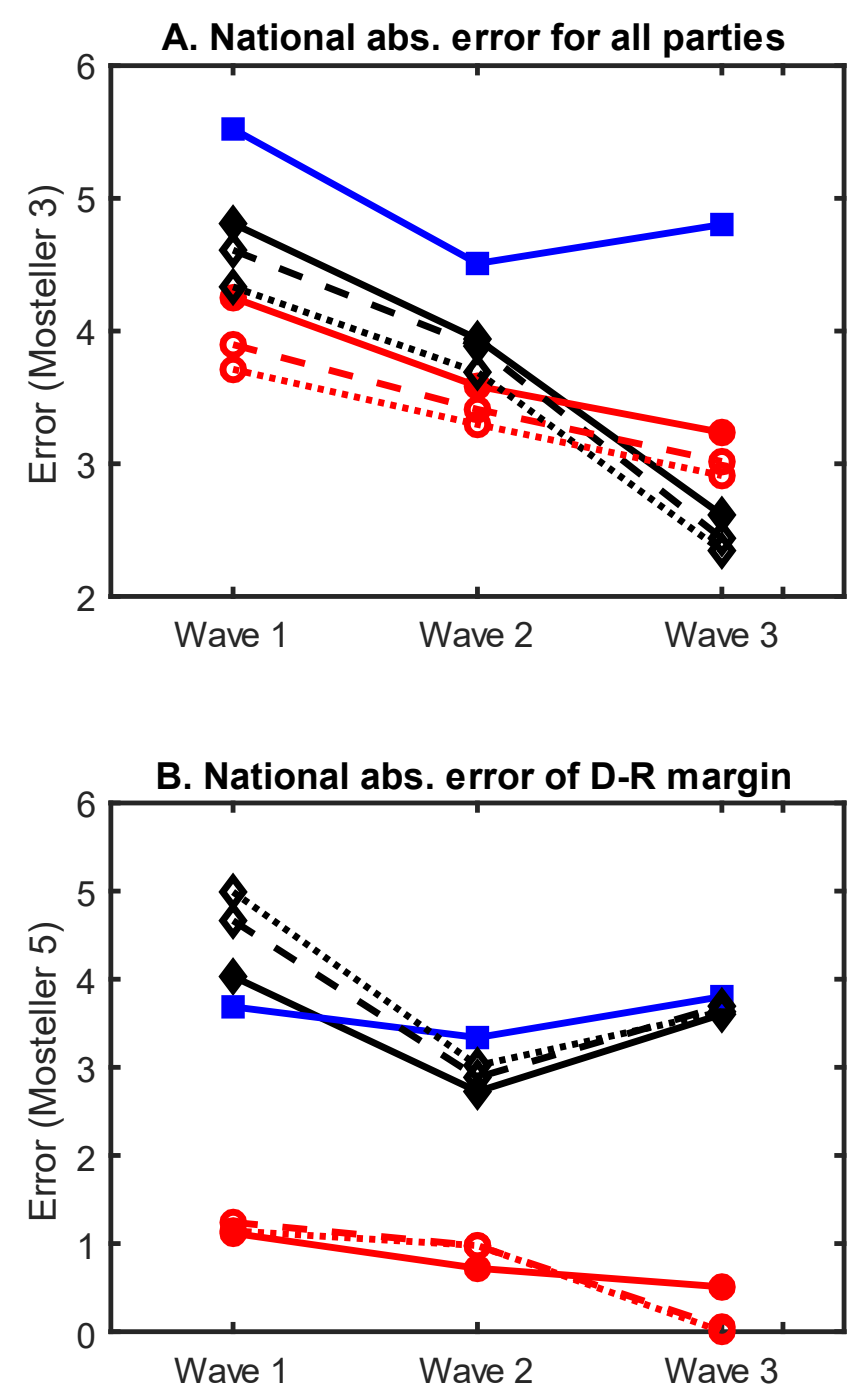

Figure 2. Error of national predictions (see Table S2 for details). Lower values are better. Panel A: The national absolute error of predicting the vote shares for all parties (Mosteller 3). Panel B: The national absolute error of predicting the Democratic and Republican vote share difference (D-R margin, Mosteller 5). 

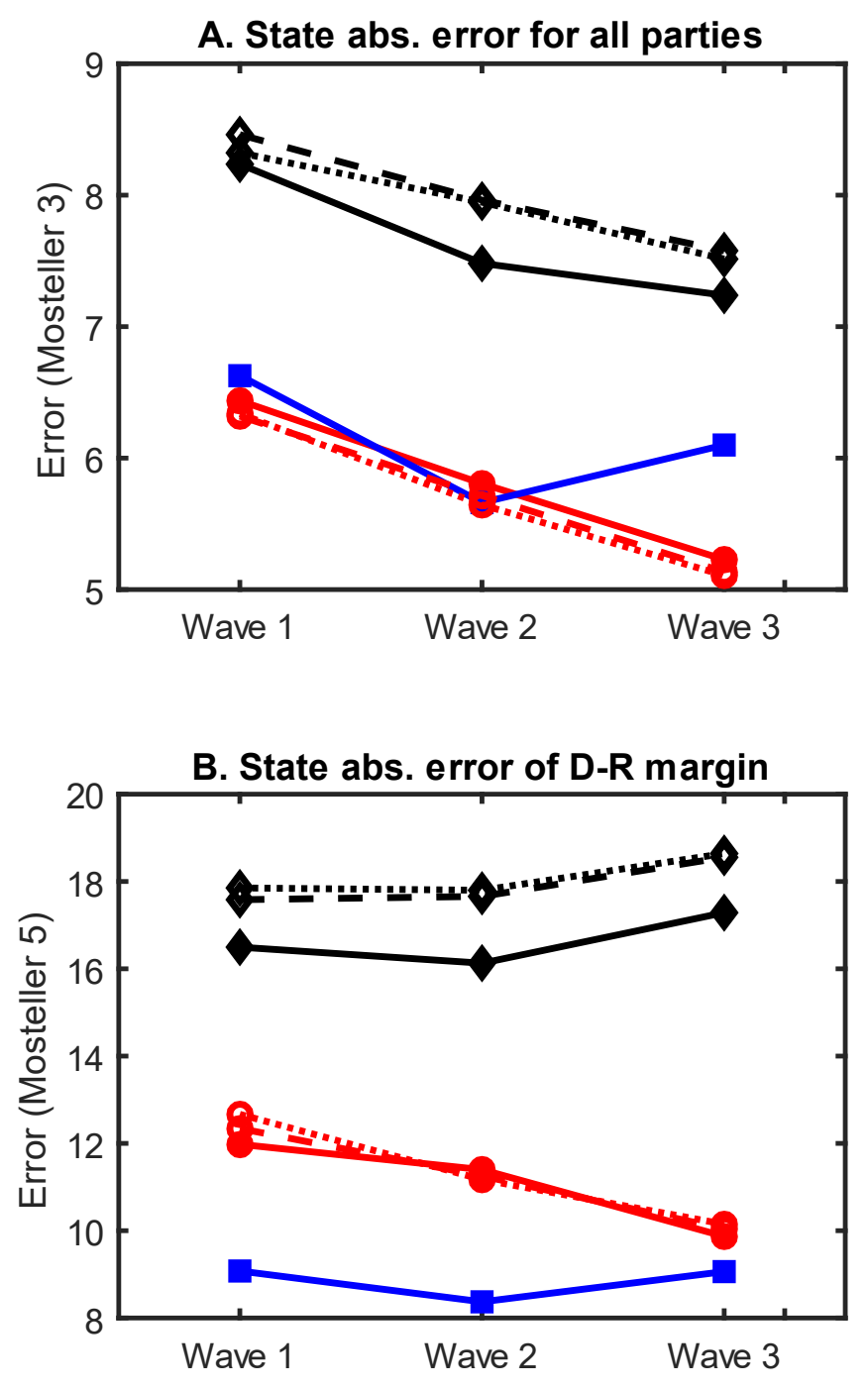

Figure 3. Average error of state predictions (see Table S3 for details). Panel A: The average state absolute error of predicting the vote shares for all parties (Mosteller 3). Panel B: The average state absolute error of predicting the Democratic and Republican vote share difference (D-R margin, Mosteller 5). Lower values are better. 


\section{Discussion}

Election predictions can be improved using novel approaches to harvest the wisdom of crowds, combined with scoring methods to detect more honest and informed respondents. These results have implications for surveys in many other domains, including public health, economic forecasts, and measurement of opinions and values.

We have presented the first demonstration that BTS scores, in the form of the original BTS or its updated version BTSD, can improve the accuracy of election polls. We provide a new result that supports BTS weighting even if there are no deceptive respondents, because it gives more weight to more informed respondents. The results also suggest further evidence for the value of wisdom of crowds approaches such as state-winner expectations and social-circle expectations (16).

The improvements due to BTS scores are dependent on the level of analysis (national or state levels) and the error measure used (error of predicted vote shares for all parties or only for the margin between two main parties). How to best design and evaluate election polls depends on the goals of the poll: In two-party systems it might be enough to be able to predict the margin of the two parties, but in countries with a multiparty system it is desirable to predict the distribution of vote shares for all parties.

On the national level, if the goal is to predict vote shares for all parties, BTS and BTSD improves predictions relative to own intentions and social-circle expectations. If the goal is to predict the margin of the two parties, BTS and BTSD do not provide as much accuracy improvements over own intentions and social-circle expectations.

On the state level, if the goal is to predict vote shares for all parties, BTS and BTSD improve the predictions for social-circle expectations but not consistently for own intentions. If the goal is to predict the margin of the two parties, neither BTS or BTSD improve the predictions from own intentions or social-circle expectations. Here, asking about state-level expectations is more accurate than any other method.

In sum, BTS and BTSD scores are most useful when applied to social-circle expectations of vote shares for all parties on the national and state levels. The scores do not improve predictions of the Democratic-Republican margin.

A possible source for the discrepancies between results for forecasting vote shares for all parties and the margin can be seen in the simulations of BTS and BTSD scores with different 
values of the weighting constant and the divergence weight (Figures S5 and S6). For all parties, the functions relating these parameters to the absolute error are well behaved, with the most error in wave 1 and the least error in wave 3. The error for the margin behaves quite differently: the ordering of the error curves is not consistent and can monotonically (or nonmonotonically) increase or decrease across survey waves. Further research is needed to understand why the D-R margin behaves so differently. Possibly, it is a result of taking the difference between two errors.

Our further results show that social-circle expectations and their BTS or BTSD variants, as well as state-winner expectations, are less sensitive to small survey sample sizes than own intentions. Our results also show that when polls are conducted well in advance of the elections, BTSD-scored social-circle expectations for all parties are most accurate on the national level. When polls are conducted close to the elections, BTSD-scored own intentions are most accurate. We have also confirmed a number of pre-registered hypotheses and have replicated the finding that social-circle expectations improve elections predictions compared to own intentions (15).

All of these results are expected to transfer to surveys of other topics beyond elections, including those related to different public opinions, health behaviors, and economic trends. In particular, our results suggest that predictions of probability distributions for different societal outcomes can be reliably improved using BTS-scored social-circle expectations.

\section{Materials and Methods}

\section{Sample}

Participants were members of the Understanding America Study (UAS) at the University of Southern California's (USC) Dornsife Center for Economic and Social Research (39). This longitudinal panel includes close to 6,000 U.S. residents, randomly selected from among all households in the United States using address-based sampling. Participants were recruited by a combination of mail, phone and web surveys. Members of recruited households who did not have Internet access were provided with tablets and Internet service.

Starting from August 22, 2018, all members of the panel were invited to answer our election poll questions. We asked the questions in three survey waves before the election, which was held on November 6, 2018. In a fourth wave after the election we asked each member which party they voted for. Wave $1(N=4,511)$ was conducted between August 22 and September 24, Wave $2(N=4,259)$ was conducted between September 14 and October 14, and Wave 3 $(N=5,038)$ was conducted between October 15 and November 5, 2018. Wave $4(N=5,099)$ was 
conducted between November 7 and December 14. Sample sizes in each wave were determined by the Center for Economic and Social Research's Understanding America Study as sufficient to provide estimates of election results within an acceptable margin of error (+/-3 p.p. at the $95 \%$ confidence level, 43). Survey weights were constructed by a raking procedure that matched the sample to national population benchmarks based on the May 2016 Current Population Survey age by sex, race/ethnicity, sex by education, and household size by income.

\section{Question texts}

Likelihood to vote: "What is the percent chance that you will vote in the 2018 election for the U.S. House of Representatives?". Own intentions: "If you do vote in the election for the U.S. House of Representatives, what is the percent chance that you will vote for the Democratic candidate? For the Republican candidate? For another party's candidate?". Social contacts definition that preceded social-circle questions: "Now we would like you to think of your friends, family, colleagues, and other acquaintances who live in your state, are at least 18 years of age, and who you have communicated with at least briefly within the last month, either faceto-face, or otherwise. We will call these people your social contacts”. Social-circle likelihood to vote: "What percentage of your social contacts are likely to vote in your state in the 2018 election for the U.S. House of Representatives?". Social-circle expectations: "Out of all your social contacts who live in your state and are likely to vote in the 2018 election, what percentage do you think will vote for a Democratic candidate? For a Republican candidate? For another party's candidates?". State-winner expectations: "Of all people who live in your state and are likely to vote, what percentage do you think will vote for a Republican candidate? For a Democratic candidate? For another party's candidate?”.

\section{Predictions from poll data}

Predictions based on own intentions. We multiplied each participant's measure of likelihood to vote multiplied by the measure of own intention to provide a basis for vote-share predictions. We then calculated the vote shares as a ratio of the average of these values and the average likelihood to vote, using a ratio mean estimator $(16,44)$. We calculated predictions on the national and state levels. On the national level, we derived predictions using two procedures. In the first procedure, we calculated ratio means for each state, and then averaged the state predictions weighted by state population size $18+$ to obtain national predictions (Table 1). In the second procedure, we obtained predictions of national vote shares by calculating ratio means for 
all participants in the national sample (Table S1). However, these latter predictions cannot be obtained for BTS-based methods which rely on state-winner expectation questions, so we focused on the first procedure of obtaining national predictions. On the state level, we calculate predictions of state vote shares as ratio means of predictions for participants in a given state.

Predictions based on social-circle expectations. We multiplied each participant's measure of social-circle likelihood to vote with the measure of social-circle expectations to provide a basis for vote-share predictions. We used these individual values in the same ways as predictions based on own intentions described above.

Predictions based on state-winner expectations. These predictions are the unaltered responses to the state-winner expectations question. The individual values are used in the same ways as predictions based on own intentions described above.

Predictions based on BTS-scored own intentions. Eq. 1 gives the equation for BTS and BTSD. The main idea behind weighting predictions with BTS is that if less honest participants receive lower-than-expected BTS scores, then weighting by scores should reduce their impact and improve survey accuracy. Furthermore, if low BTS scores arise from a subset of less informed respondents whose answers are drawn from a prior distribution, while higher-scored answers are drawn from a true (posterior) distribution of voting intentions, then small BTS weighting always improves accuracy compared to the unweighted average (proof in the Appendix). Without knowing the percentage of less informed respondents, however, one cannot compute the optimal BTS weight. Weighting involves an element of judgment, informed by simulations or past experience with similar surveys. Our pre-registered weight $(0.25)$ was suggested by simulations with $10 \%$ less informed respondents.

The following steps were performed to calculate predictions based on Bayesian Truth Serum own intentions: 1 ) add $1 \%$ to own intentions and to state-winner expectations (both multiplied by own likelihood to vote to correspond to the baseline predictions based on own intentions) for each of the three questions (about Republican, Democratic, and Other candidates) and divide by $1.03,2)$ compute the mean of the measure of own intentions ( $\bar{x}$ in Eq. 1), 3) take the natural logarithm of the state-winner expectations, 4) compute the means of 3 for each state, 5) exponentiate 4 yielding the geometric mean ( $\bar{y}$ in Eq. 1), 6) compute the Information score and the Prediction score for each participant based on 2 and 5, 7) add the Information scores and the Prediction scores to get the BTS scores, 8) exponentiate 7 multiplied by a constant (in the 
preregistered analyses it is equal to 0.25 ) to get the unnormalized BTS weights, 9) compute the mean of 8 for each state, 10) divide 8 by 9 to get state-normalized BTS weights, 11) multiply the weight in 10 with own intentions, and finally, 12) to get the predictions based on BTS own intentions the values in 11 are weighted with likelihood to vote in the same way as for predictions based on own intentions. The computation of the BTSD involves another step: to calculate the divergence score we first predict state-winner expectations that participants should provide if they were similar to other participants in their state who had the same own intentions. To do this we fit a regression equation $\bar{y}_{k}^{r}=x_{k}^{r}+e$, separately for each state $\left(x_{k}^{r}\right.$ is the is the answer to the own intention question). If there were less than 20 participants in a state, we impute $\bar{y}_{k}^{r}$ from the same regression fitted on the whole sample. Then, in step 7 above, we also add the divergence score weighted by the parameter $\lambda$, which we set to 1 . This is the simplest assumption that produces equal weights to all three scores.

Predictions based on BTS-scored social-circle expectations. Computed in the same way as predictions based on BTS own intentions, but with own intentions and own likelihood to vote substituted by social-circle expectations and social-circle likelihood to vote.

\section{Weighting of predictions}

When calculating state and national averages of the predictions, individual responses were weighted by poststratification weights that align the survey distributions to benchmarks for region, age by sex, education, income, and race/ethnicity. For a description of how weights are constructed, see (43). When calculating BTS indices, poststratification weights are also applied in step (9) above.

\section{Election results}

We used the national election results collected by The Cook Reports Election tracker (45). At the national level, we used the national popular vote as the benchmark. This benchmark was used in two kinds of comparisons, one based on predictions from the whole sample of participants and one based on the state-average predictions. At the state level, we used the state's popular votes as the benchmark. We first computed the errors in predicting each states' popular vote and then averaged all values to get an overall measure of state-level accuracy. 


\section{References}

1. National Health Interview Survey Available at: https://www.cdc.gov/nchs/nhis/index.htm.

2. Armantier O, Bruine de Bruin W, Potter S, Topa G, van der Klaauw, W. Zafar B (2013) Measuring inflation expectations. Annu Rev Econom 5:273-301.

3. Surveys of Consumers University of Michigan Available at: https://data.sca.isr.umich.edu/.

4. World Value Survey Available at: http://www.worldvaluessurvey.org/wvs.jsp.

5. Pew (2019) Election polling. Available at: https://www.pewresearch.org/methods/u-ssurvey-research/election-polling/.

6. Jennings W, Wlezien C (2018) Election polling errors across time and space. Nat Hum Behav 2(4):276-283.

7. Kennedy C, et al. (2018) An evaluation of the 2016 election polls in the united states. Public Opin Q 82(1):1-33.

8. Lewis-Beck MS, Tien C (1999) Voters as forecasters: A micromodel of election prediction. Int J Forecast 15(2):175-184.

9. Murr AE (2016) The wisdom of crowds: What do citizens forecast for the 2015 British General Election? Elect Stud 41:283-288.

10. Graefe A (2014) Accuracy of vote expectation surveys in forecasting elections. Public Opin Q 78(S1):204-232.

11. Bruine de Bruin W, Downs JS, Murray P, Fischhoff B (2010) Can female adolescents tell whether they will test positive for chlamydia infection? Med Decis Mak 30(2):189-193.

12. Bruine de Bruin W, Parker AM, Fischhoff B (2007) Can adolescents predict significant life events? J Adolesc Heal 41(2):208-210.

13. de Bruin WB, Parker AM, Galesic M, Vardavas R (2019) Reports of social circles' and own vaccination behavior: A national longitudinal survey. Heal Psychol. doi:10.1037/hea0000771.

14. Hurd MD, McGarry K (2002) The predictive validity of subjective probabilities of survival. Econ J 112(482):966-985.

15. Rothschild DM, Wolfers J (2011) Forecasting elections: Voter intentions versus xxpectations. SSRN Electron J. doi:10.2139/ssrn.1884644.

16. Galesic M, et al. (2018) Asking about social circles improves election predictions. Nat 
Hum Behav 2(3):187-193.

17. Irwin GA, Van Holsteyn JJM (2002) According to the polls: The influence of opinion polls on expectations. Public Opin Q 66(1):92-104.

18. Bruine de Bruin W, Galesic M, Strandberg T, de Bresser J, van Soest A (2019) Asking about social circles improves election predictions: Replication in the Netherlands and Sweden.

19. Galesic M, Olsson H, Rieskamp J (2012) Social sampling explains apparent biases in judgments of social environments. Psychol Sci 23(12):1515-1523.

20. Galesic M, Olsson H, Rieskamp J (2018) A sampling model of social judgment. Psychol Rev 125(3):363-390.

21. Blais A, Gidengil E, Fournier P, Nevitte N, Hicks BM (2008) Measuring expectations: Comparing alternative approaches. Elect Stud 27(2):337-343.

22. Huckfeldt RR, Sprague J (1995) Citizens, politics and social communication: Information and influence in an election campaign (Cambridge University Press, New York).

23. Santoro L, Beck PA (2017) Social networks and vote choice. The Oxford Handbook of Political Networks, eds Victor JN, Montgomery AH, Lubell M (Oxford University Press, Oxford, UK), pp 383-406.

24. Sinclair B (2012) The social citizen: Peer networks and political behavior (University of Chicago Press, Chicago).

25. Prelec D, Seung HS, McCoy J (2017) A solution to the single-question crowd wisdom problem. Nature 541(7638):532-535.

26. Curran PG (2016) Methods for the detection of carelessly invalid responses in survey data. J Exp Soc Psychol 66:4-19.

27. Mellers B, et al. (2015) Identifying and cultivating superforecasters as a method of improving probabilistic predictions. Perspect Psychol Sci 10(3):267-281.

28. Rosenfeld B, Imai K, Shapiro JN (2016) An empirical validation study of popular survey methodologies for sensitive questions. Am J Pol Sci 60(3):783-802.

29. Faltings B, Radanovic G (2017) Game theory for data science: Eliciting truthful information doi:10.2200/S00788ED1V01Y201707AIM035.

30. Prelec D (2004) A Bayesian truth serum for subjective data. Science (80- ) 306(5695):462-466. 
31. John LK, Loewenstein G, Prelec D (2012) Measuring the prevalence of questionable research practices with incentives for truth telling. Psychol Sci 23(5):524-532.

32. Baillon A (2017) Bayesian markets to elicit private information. Proc Natl Acad Sci U S A 114(30):7958-7962.

33. Witkowski J, Parkes DC (2012) A robust bayesian truth serum for small populations. Proc Twenty-Sixth AAAI Conf Artif Intell:1492-1498.

34. Radanovic G, Faltings B (2013) A robust Bayesian truth serum for non-binary signals. Proceedings of the 27th AAAI Conference on Artificial Intelligence, AAAI 2013, pp 833839.

35. Radanovic G, Faltings B (2014) Incentives for truthful information elicitation of continuous signals. Proceedings of the National Conference on Artificial Intelligence, pp $770-776$.

36. Howie PJ, Wang Y, Tsai J (2010) Predicting new product adoption using Bayesian truth serum. J Med Mark 11(1):6-16.

37. Radas S, Prelec D (2019) Uncovering Reliable Respondents: An Application of Bayesian Truth Serum.

38. Weaver R, Prelec D (2013) Creating truth-telling incentives with the Bayesian truth serum. J Mark Res 50(3):289-302.

39. Understanding America Study Available at: https://uasdata.usc.edu/index.php.

40. Olsson H, Galesic M, Bruine De Bruin W, Prelec D (2018) Predicting the 2018 United States House of Representatives elections based on social-circle expectations, state-winner expectations, and Bayesian Truth Serum (BTS). Available at: https://osf.io/a5fpb/.

41. Mosteller F (1949) Measuring the error. The Pre-Election Polls of 1948, eds Mosteller F, Hyman H, McCarthy PJ, Marks ES, Truman DB (Social Science Research Council, New York). Available at: https://academic.oup.com/poq/article-lookup/doi/10.1093/poq/nfi044.

42. Martin EA, Traugott MW, Kennedy C (2005) A review and proposal for a new measure of poll accuracy. Public Opin $Q$ 69(3):342-369.

43. Understanding America Study: Weighting procedures Available at: https://uasdata.usc.edu/addons/documentation/UAS Weighting Procedures.pdf.

44. Gutsche TL, Kapteyn A, Meijer E, Weerman B (2014) The RAND continuous 2012 presidential election poll. Public Opin $Q$ 78(S1):233-254. 
45. 2018 House Popular Vote Tracker Available at:

https://cookpolitical.com/analysis/house/house-charts/2018-house-popular-vote-tracker.

46. Cvitanić J, Prelec D, Radas S, Šikić H (2019) Incentive compatible surveys via posterior probabilities. SIAM Theory Probab Its Appl. 


\section{Appendix}

The Proposition in the main text builds on the theoretical results for Bayesian truth serum (30) and shares some key assumptions. We first review these and then provide a proof of the Proposition.

BTS scoring (30) was introduced to provide incentives for honest responding to a multiple-choice question (MCQ), in a setting where honesty cannot be independently verified. E ach $\mathrm{r}$ espondent p rovides a primary and a secondary input. The primary input is a selection of one answer from the list of $m$ possible answers to the MCQ; the secondary input is a prediction in percentages of the distribution of answers (i.e., the primary inputs) to the MCQ.

Denote the answer selected by respondent $r$ by indicator (dummy) variables $x_{k}^{r}, k=1, \ldots, m$, with $x_{k}^{r}=1$ if respondent $r$ selected answer $k$ and zero otherwise. Similarly, denote by $y_{k}^{r}, k=1, \ldots, m, r$ 's prediction

of the percent of respondents selecting answer $k$. The BTS score for respondent $r$ is a function of $x_{k}^{r}$ and $y_{k}^{r}$ and two sample statistics: the actual percent selecting answer $k, \bar{x}_{k}=\frac{1}{n} \sum_{r} x_{k}^{r}$, and the geometric mean of all predictions, $\bar{y}_{k}=\exp \left(\frac{1}{n} \sum_{r} \log y_{k}^{r}\right)$ :

$$
u^{r}=\sum_{k=1}^{m} x_{k}^{r} \log \frac{\bar{x}_{k}}{\bar{y}_{k}}+\sum_{k=1}^{m} \bar{x}_{k} \log \frac{y_{k}^{r}}{\bar{x}_{k}}
$$

The theoretical properties of BTS (stated below) rest on several assumptions. First, the sample is large, strictly speaking countably infinite. Second, each respondent has a unique true answer to the question, called their 'type' $t^{r} \in\{1, . ., m\}$. An honest answer reveals the type, $x_{k}^{r}=1 \Longleftrightarrow t^{r}=k$. The actual distribution of types is given by a probability vector $\omega=\left(\omega_{1}, . ., \omega_{m}\right), \omega_{i} \geq 0, \sum_{i=1}^{m} \omega_{i}=1$. If all $n$ respondents are honest, we would observe this distribution in the large sample limit:

$$
\bar{x}_{k}=\lim _{n \rightarrow \infty} \frac{1}{n} \sum_{r} x_{k}^{r}=\omega_{k}
$$


Third, respondents derive beliefs about the type distribution by updating from a common prior $p\left(\omega_{1}, \ldots, \omega_{m}\right)$, and treating their type as a 'sample-of-one.' That is, a respondent with type $t^{r}=i$ computes a posterior distribution $p\left(\omega_{1}, \ldots, \omega_{m} \mid t^{r}=i\right)$ by applying Bayes' rule:

$$
p\left(\omega_{1}, \ldots, \omega_{m} \mid t^{r}=i\right)=\frac{p\left(t^{r}=i \mid \omega_{1}, \ldots, \omega_{m}\right) p\left(\omega_{1}, \ldots, \omega_{m}\right)}{p\left(t^{r}=i\right)}=\frac{\omega_{i}}{\pi_{i}} p\left(\omega_{1}, \ldots, \omega_{m}\right)
$$

with $\pi_{i}=p\left(t^{r}=i\right)$ denoting the prior probability of type $i$ (i.e., the expected value of the prior on coordinate $i$ ), while the probability $p\left(t^{r}=i \mid \omega_{1}, \ldots, \omega_{m}\right)$ that respondent $r$ is of type $i$ is nothing but $\omega_{i}$, the actual frequency of type $i$-s in the population. An honest prediction of percent of types $k$ by a respondent of type $i$ is then the expected value of the posterior,

$$
y_{k}^{r}=E\left(\omega_{k} \mid t^{r}=i\right)=\int_{\omega} \omega_{k} p\left(\omega_{1}, \ldots, \omega_{m} \mid t^{r}=i\right)
$$

The first term in (1) is the information score. If all respondents' excluding respondent $r$ are honest, then an honest primary answer by respondent $r$ maximizes his expected or 'ex-ante' information score:

$$
E\left(\log \frac{\bar{x}_{k}}{\bar{y}_{k}} \mid t^{r}=k\right)>E\left(\log \frac{\bar{x}_{j}}{\bar{y}_{j}} \mid t^{r}=k\right)
$$

for any $j \neq k$. The expectation on the left is computed for an honest answer, $x_{k}^{r}=1$, implying $\sum_{i=1}^{m} x_{i}^{r} \log \frac{\bar{x}_{i}}{\bar{y}_{i}}=$ $\log \frac{\bar{x}_{k}}{\bar{y}_{k}}$; the expectation on the right is computed for a dishonest response, $x_{j}^{r}=1$, implying $\sum_{i=1}^{m} x_{i}^{r} \log \frac{\bar{x}_{i}}{\bar{y}_{i}}=$ $\log \frac{\bar{x}_{j}}{\bar{y}_{j}}$. Because the second term in (1), the prediction score, does not depend on $x_{k}^{r}$ in a large sample, honest answers also maximize the BTS score, $u^{r}$.

This property of BTS generates truth-telling incentives; however, the proof of the Proposition in the main text does not involve it. Instead, the proof relies on Theorem 2 in (30), which states that the eqiulibrium BTS score for respondent with type $t^{r}=k$, which we denote by $u^{r}\left(t^{r}=k ; \bar{x}_{1}, . ., \bar{x}_{m}\right)$, is asymptotically equal to the $\log$ probability that respondent with type $t^{r}=k$ assigns to the ex-post distribution of answers $\left(\bar{x}_{1}, . ., \bar{x}_{m}\right)$, plus a budget-balancing constant $C$ :

$$
\lim _{n \rightarrow \infty} u^{r}\left(t^{r}=k ; \bar{x}_{1}, . ., \bar{x}_{m}\right)=\log p\left(\bar{x}_{1}, . ., \bar{x}_{m} \mid t^{r}=k\right)+C
$$

Respondents with higher BTS scores assign a higher probability on the observed distribution of answers. 
Theorem 2 in (30) states this result in terms of the type distribution $\left(\omega_{1}, \ldots, \omega_{m}\right)$ rather than the answer distribution $x^{-}, \ldots, x_{m}^{-}$, but the two distributions are identical in equilibrium (equation (2)). (46) provides a proof in terms of the answer distribution.

In the Proposition in the main text, this equivalence no longer holds; instead, it is assumed that a fraction of respondents are drawn from the actual distribution $\left(\omega_{1}, \ldots, \omega_{m}\right)$ while the remainder $(\varepsilon)$ are drawn from the prior $\left(\pi_{1}, \ldots, \pi_{m}\right)$. Even if everyone is honest, the observed distribution will be a mixture of the actual and prior distributions:

$$
\bar{x}_{k}=(1-\varepsilon) \omega_{k}+\varepsilon \pi_{k} \quad k=1, \ldots, m,
$$

We assume also that this fact is common knowledge among all respondents, and is reflected in their predictions $y_{k}^{r}$.

In the voting survey context, the actual distribution is the distribution of votes by individuals in the sample on election day. The assumption here is that a fraction $(1-\varepsilon)$ correctly predicts and reports their future vote while the rest distribute themselves acording to the prior. The goal of the reweighting is to obtain an estimate that is closer to the distribution $\left(\omega_{1}, \ldots, \omega_{m}\right)$ that matches actual voting behavior of people in the sample.

Proposition. Let $\bar{x}_{k}(\beta)$ denote the average of answers with respondents weighted by their BTS scores:

$$
\bar{x}_{k}(\beta)=\frac{\sum_{r} \exp \left(\beta u^{r}\right) x_{k}^{r}}{\sum_{j=1}^{m} \sum_{r} \exp \left(\beta u^{r}\right) x_{j}^{r}}
$$

If a fraction $\varepsilon>0$ of respondents are sampled from the prior, while the remaining $(1-\varepsilon)$ are sampledfrom the actual distribution $\omega$, then for all $\beta$ in an interval: $0<\beta<\beta^{\circ}, \bar{x}_{k}(\beta)$ will be closer to the actual distribution $\omega_{k}$ than the unweighted average $\bar{x}_{k}(\beta=0)$.

Proof. Without loss of generality, order answers so that answer $k$ is underestimated relative to answer $j$. Given equation (4) this ordering can be expressed in three equivalent ways:

$$
k>j \Longrightarrow \frac{\omega_{k}}{\pi_{k}} \geq \frac{\omega_{j}}{\pi_{j}} \Longleftrightarrow \frac{\bar{x}_{k}}{\pi_{k}} \geq \frac{\bar{x}_{j}}{\pi_{j}} \Longleftrightarrow \frac{\omega_{k}}{\bar{x}_{k}} \geq \frac{\omega_{j}}{\bar{x}_{j}}
$$

Note also that the ordering is unique except for ties. From equation (3), we see that the score difference of 
two respondents equals the log ratio of probabilities they assign on the observed distribution of answers:

$$
\begin{aligned}
u^{r}\left(t^{r}=k ; \bar{x}_{1}, . ., \bar{x}_{m}\right)-u^{s}\left(t^{s}=j ; \bar{x}_{1}, . ., \bar{x}_{m}\right) & =\log \frac{p\left(\bar{x}_{1}, . ., \bar{x}_{m} \mid t^{r}=k\right)}{p\left(\bar{x}_{1}, . ., \bar{x}_{m} \mid t^{s}=j\right)} \\
& =\log \frac{p\left(t^{r}=k \mid \bar{x}_{1}, . ., \bar{x}_{m}\right) p\left(t^{s}=j\right)}{p\left(t^{s}=j \mid \bar{x}_{1}, . ., \bar{x}_{m}\right) p\left(t^{r}=k\right)} \\
& =\log \frac{\bar{x}_{k} \pi_{j}}{\bar{x}_{j} \pi_{k}}
\end{aligned}
$$

which implies:

$$
\frac{\exp \left(u^{r}\left(t^{r}=k ; \bar{x}_{1}, . ., \bar{x}_{m}\right)\right)}{\exp \left(u^{s}\left(t^{s}=j ; \bar{x}_{1}, . ., \bar{x}_{m}\right)\right)}=\frac{\bar{x}_{k} \pi_{j}}{\bar{x}_{j} \pi_{k}}
$$

From (1), we may express the ratio of BTS-weighted answer percentages as:

$$
\begin{aligned}
\frac{\bar{x}_{k}(\beta)}{\bar{x}_{j}(\beta)} & =\frac{\sum_{r} \exp \left(\beta u^{r}\right) x_{k}^{r}}{\sum_{s} \exp \left(\beta u^{s}\right) x_{j}^{s}} \\
& =\frac{\exp \left(\beta u^{r}\left(t^{r}=k ; \bar{x}_{1}, . ., \bar{x}_{m}\right)\right) \bar{x}_{k}}{\exp \left(\beta u^{s}\left(t^{s}=j ; \bar{x}_{1}, . ., \bar{x}_{m}\right)\right) \bar{x}_{j}} \\
& =\left(\frac{\bar{x}_{k} \pi_{j}}{\bar{x}_{j} \pi_{k}}\right) \frac{\bar{x}_{k}}{\bar{x}_{j}} \\
& >\frac{\bar{x}_{k}}{\bar{x}_{j}}
\end{aligned}
$$

as $\left(\frac{\bar{x}_{k} \pi_{j}}{\bar{x}_{j} \pi_{k}}\right)^{\beta}>1$ by the ordering assumption, $\bar{x}_{k} / \pi_{k}>\bar{x}_{j} / \pi_{j}$. (The second line above exploits the fact that respondents selecting the same answer receive the same score, i.e., $x_{k}^{r}=x_{k}^{s}=1 \Longrightarrow u^{r}=u^{s}$.) Clearly, the ratio $\bar{x}_{k}(\beta) / \bar{x}_{j}(\beta)$ is a continuous, monotonic function of $\beta$ without an upper bound. Therefore, there is a unique value $\beta^{k j}$ that recovers the true ratio $\omega_{k} / \omega_{j}$ :

$$
\frac{\omega_{k}}{\omega_{j}}=\frac{\bar{x}_{k}\left(\beta^{k j}\right)}{\bar{x}_{j}\left(\beta^{k j}\right)}>\frac{\bar{x}_{k}}{\bar{x}_{j}}
$$

Furthermore, for all values $0<\beta<\beta^{k j}$, the ratio $\bar{x}_{k}(\beta) / \bar{x}_{j}(\beta)$ will be closer to the actual ratio $\omega_{k} / \omega_{j}$ than the unweighted ratio $\bar{x}_{k} / \bar{x}_{j}$. Setting $\beta^{\circ}=\min _{k, j}\left\{\beta^{k j}\right\}$ implies that $0<\beta<\beta^{\circ}$ ensures that the weighted distribution is closer the the actual for any pair of anwers, proving the Proposition.

Remark 1. Respondent credibility can be modeled in two ways within the general BTS framework. The approach developed here locates differences at the level of the sampling process, rather than characteristics 
of individuals. It is as if respondents' stated answers, i.e., voting intentions or social circle reports, are generated by each tossing a biased coin (or by a more complex randomizing device). There are two coins active - a true coin and a fake coin, whose bias is consistent with the prior. The researcher would like to estimate the bias of the true coin; the fake coin is noise. Respondents observe and report the result of their toss, and they know that two coins exist, but they do not know whether their toss is coming from the true coin or the fake coin. For example, the fake 'prior' coin might represent 'stale information,' with respondents answering based on their behavior in the previous election, or their social circle information from that time period. The bias of the fake coin (i.e., results of the previous election) is common knowledge, while the bias of the true coin (i.e., results of the next election) is of course not known by any respondent.

An alternative approach would be to model individual differences in information or expertise explicitly. This was developed in (37) in the context of market research surveys. The assumption there was that less informed respondents could not evaluate the quality or appeal of new product concept from the materials presented in the survey. Consequently, their predictions of purchase intent by other respondents would be less accurate, and they know this to be the case. In terms of the above coin model, respondents know the nature of their coin (true or fake). The difference in prediction accuracy allows one to identify the knoweldgeable respondents, and project to the market using only the knowledgeables' purchase intent data.

Remark 2. In the main text, we have added a third term to obtain the extended BTSD equation. However, the third term has value zero if everyone responds honestly, and plays no role in the proof of the Proposition. Let $\bar{y}_{k}^{r}$ denote the (geometric) average prediction of respondents selecting the same answer (excluding respondent $r)$ :

$$
\bar{y}_{k}^{r}=\exp \left(\frac{\sum_{s \neq r} x_{k}^{r} \log y_{k}^{s}}{\sum_{s \neq r} x_{k}^{r} x_{k}^{s}}\right)
$$

Note that the product $x_{k}^{r} x_{k}^{s}$ identifies respondents $s$ who selected the same answer as respondent $r$. The extended BTS score is then:

$$
u^{r}=\sum_{k=1}^{m} x_{k}^{r} \log \frac{\bar{x}_{k}}{\bar{y}_{k}}+\sum_{k=1}^{m} \bar{x}_{k} \log \frac{y_{k}^{r}}{\bar{x}_{k}}+\lambda \sum_{k=1}^{m} \bar{y}_{k}^{r} \log \frac{\bar{y}_{k}^{r}}{y_{k}^{r}}
$$

Under our assumptions, respondents that give the same answer will also give the same prediction, hence $y_{k}^{r}=\bar{y}_{k}^{r}$ and $\log \frac{\bar{y}_{k}^{r}}{y_{k}^{r}}=0$. 


\section{Supplementary Information}

\section{Harvesting the wisdom of crowds for election predictions using the Bayesian Truth Serum}

Henrik Olsson, Wändi Bruine de Bruin, Mirta Galesic, \& Drazen Prelec

\section{Contents}

\section{Tables for additional analyses}

National predictions based on the whole survey sample (Table S1), errors of national predictions (Table S2), average error of state predictions (Table S3), and predictions including only states with more than 30 survey participants (Table S4)

\section{Figures for additional analyses}

Error differences between different methods for predicting vote shares for all parties (Mosteller 3) as a function of sample size in each state (Figure S1), error differences between different methods for predicting the D-R margin (Mosteller 5) as a function of sample size in each state (Figure S2), error of different methods for predicting vote shares for all parties (Mosteller 3) for different sample sizes (Figure S3), error for predicting the D-R margin (Mosteller 5) for different sample sizes (Figure S4).

\section{Simulation results}

Simulations with different values of the weighting constant (Figure S5) and the divergence weight (Figure S6)

\section{Preregistered hypotheses}

Description and results for preregistered hypotheses.

\section{Predicting individual voting behavior}

Results of linear regressions predicting reported voting behavior from own intentions, social circle expectations, and state expectations (Tables S5-S7) 


\section{Tables for additional analyses}

Table S1

National predictions based on the whole survey sample

\begin{tabular}{|c|c|c|c|c|}
\hline & Election results & Own intentions & $\begin{array}{l}\text { Social-circle } \\
\text { expectations }\end{array}$ & $\begin{array}{l}\text { State-winner } \\
\text { expectations }\end{array}$ \\
\hline \multicolumn{5}{|l|}{ Wave 1 (Aug $22-$ Sep 11) } \\
\hline Republicans & 44.8 & 39.8 & 42.9 & 43.6 \\
\hline Democrats & 53.4 & 51.5 & 49.0 & 46.5 \\
\hline Other & 1.7 & 8.7 & 8.1 & 9.9 \\
\hline Democrats-Republicans & 8.6 & 11.7 & 6.1 & 3.0 \\
\hline RMSE for all parties & & 5.1 & $\underline{4.6}$ & 6.2 \\
\hline Error of D-R margin & & 3.1 & $\underline{-2.5}$ & -5.6 \\
\hline \multicolumn{5}{|l|}{ Wave $2($ Sep $14-$ Oct 4$)$} \\
\hline Republicans & 44.8 & 41.2 & 43.1 & 43.7 \\
\hline Democrats & 53.4 & 51.3 & 49.9 & 47.9 \\
\hline Other & 1.7 & 7.5 & 7.1 & 8.4 \\
\hline Democrats-Republicans & 8.6 & 10.1 & 6.8 & 4.2 \\
\hline RMSE for all parties & & 4.1 & $\underline{3.8}$ & 5.0 \\
\hline Error of D-R margin & & $\underline{1.5}$ & $-\overline{1.8}$ & -4.3 \\
\hline \multicolumn{5}{|l|}{ Wave 3 (Oct 15 - Nov 5) } \\
\hline Republicans & 44.8 & 41.3 & 42.3 & 43.6 \\
\hline Democrats & 53.4 & 53.2 & 51.3 & 47.6 \\
\hline Other & 1.7 & 5.5 & 6.4 & 8.8 \\
\hline Democrats-Republicans & 8.6 & 11.9 & 9.0 & 4.0 \\
\hline RMSE for all parties & & $\underline{\mathbf{3 . 0}}$ & 3.3 & 5.3 \\
\hline Error of D-R margin & & 3.3 & $\underline{0.4}$ & -4.6 \\
\hline
\end{tabular}

Note. Bold underline indicate predictions with lowest error within the survey. Smallest errors within the USC survey are shown in underlined bold.

Note that predictions based on the Bayesian Truth Serum (BTS) are only possible on the level of each state. Consequently, BTS-based national predictions can be calculated only as weighted average of state results (provided in Tables 1, S2-S4; and in Figures 1 and 2). 
Table S2

Errors of national predictions (calculated as weighted average of state predictions, see Table 1)

\begin{tabular}{|c|c|c|c|c|c|c|c|}
\hline & $\begin{array}{r}\text { Own } \\
\text { intentions } \\
\end{array}$ & $\begin{array}{l}\text { Social-circle } \\
\text { expectations }\end{array}$ & $\begin{array}{l}\text { State-winner } \\
\text { expectations }\end{array}$ & $\begin{array}{l}\text { BTS own } \\
\text { intentions }\end{array}$ & $\begin{array}{r}\text { BTS social- } \\
\text { circle } \\
\text { expectations } \\
\end{array}$ & $\begin{array}{r}\text { BTSD own } \\
\text { intentions } \\
\end{array}$ & $\begin{array}{r}\text { BTSD } \\
\text { social-circle } \\
\text { expectations } \\
\end{array}$ \\
\hline \multicolumn{8}{|l|}{ Wave 1 (Aug 22 - Sep 11) } \\
\hline Average absolute error for all parties & 4.8 & 4.3 & 5.5 & 4.6 & 3.9 & 4.3 & $\underline{3.7}$ \\
\hline $\begin{array}{l}\text { Average absolute error of D-R margin } \\
\text { Wave } 2 \text { (Sep } 14 \text { - Oct } 4)\end{array}$ & 4.0 & $\underline{-1.12}$ & -3.7 & 4.7 & -1.2 & 5.0 & -1.14 \\
\hline Average absolute error for all parties & 3.9 & 3.6 & 4.5 & 3.9 & 3.4 & 3.7 & $\underline{3.3}$ \\
\hline \multicolumn{8}{|l|}{ Wave 3 (Oct 15 - Nov 5) } \\
\hline Average absolute error for all parties & 2.6 & 3.2 & 4.8 & 2.4 & 3.0 & $\underline{2.3}$ & 2.9 \\
\hline Average absolute error of D-R margin & 3.6 & 0.5 & -3.8 & 3.7 & 0.1 & 3.6 & $\underline{0.0}$ \\
\hline
\end{tabular}

Note. BTS = Bayesian Truth Serum. BTSD = Bayesian Truth Serum with divergence score (see Eq. 1). Lower values are better. Bold underline indicates the lowest errors within the USC survey. This data is shown in Figure 1. 
Table S3

Average error of state predictions

\begin{tabular}{|c|c|c|c|c|c|c|c|}
\hline & $\begin{array}{r}\text { Own } \\
\text { intentions }\end{array}$ & $\begin{array}{l}\text { Social-circle } \\
\text { expectations }\end{array}$ & $\begin{array}{l}\text { State-winner } \\
\text { expectations }\end{array}$ & $\begin{array}{l}\text { BTS own } \\
\text { intentions }\end{array}$ & $\begin{array}{r}\text { BTS social- } \\
\text { circle } \\
\text { expectations }\end{array}$ & $\begin{array}{r}\text { BTSD own } \\
\text { intentions }\end{array}$ & $\begin{array}{r}\text { BTSD } \\
\text { social-circle } \\
\text { expectations } \\
\end{array}$ \\
\hline \multicolumn{8}{|l|}{ Wave 1 (Aug 22 - Sep 11) } \\
\hline Average absolute error for all parties & 7.6 & 6.3 & 6.8 & 7.7 & 6.2 & 7.5 & $\underline{6.1}$ \\
\hline \multicolumn{8}{|l|}{ Wave $2($ Sep $14-$ Oct 4$)$} \\
\hline Average absolute error for all parties & 7.0 & 5.8 & 6.0 & 7.3 & 5.7 & 7.2 & $\underline{5.6}$ \\
\hline $\begin{array}{l}\text { Average absolute error of D-R margin } \\
\text { Wave } 3 \text { (Oct } 15 \text { - Nov } 5 \text { ) }\end{array}$ & 13.8 & 10.5 & $\underline{8.4}$ & 15.0 & 10.5 & 15.1 & 10.5 \\
\hline Average absolute error for all parties & 7.0 & 5.8 & 6.0 & 6.7 & $\underline{4.9}$ & 6.6 & $\underline{4.9}$ \\
\hline Average absolute error of D-R margin & 14.2 & 9.0 & 8.4 & 15.1 & 9.0 & 15.3 & 9.0 \\
\hline
\end{tabular}

Note. BTS = Bayesian Truth Serum. BTSD = Bayesian Truth Serum with divergence score (see Eq. 1 and Appendix). Lower values are better.

Bold underline indicates the lowest errors within the USC survey. This data is shown in Figure 2. 
Predictions including only states with more than 30 survey participants (Wave 3; compare with Tables 1, S2, and S3)

\begin{tabular}{|c|c|c|c|c|c|c|c|c|}
\hline & $\begin{array}{r}\text { Election } \\
\text { results } \\
\end{array}$ & $\begin{array}{r}\text { Own } \\
\text { intentions }\end{array}$ & $\begin{array}{r}\text { Social- } \\
\text { circle } \\
\text { expectatio } \\
\mathrm{ns} \\
\end{array}$ & $\begin{array}{r}\text { State- } \\
\text { winner } \\
\text { expectatio } \\
n s \\
\end{array}$ & $\begin{array}{l}\text { BTS own } \\
\text { intentions }\end{array}$ & $\begin{array}{r}\text { BTS } \\
\text { social- } \\
\text { circle } \\
\text { expectatio } \\
\text { ns } \\
\end{array}$ & $\begin{array}{r}\text { BTSD own } \\
\text { intentions } \\
\end{array}$ & $\begin{array}{r}\text { BTSD } \\
\text { social- } \\
\text { circle } \\
\text { expectatio } \\
\text { ns } \\
\end{array}$ \\
\hline \multicolumn{9}{|l|}{ National predictions } \\
\hline Republicans & 44.8 & 41.0 & 41.6 & 42.8 & 41.1 & 42.0 & $\underline{41.2}$ & 42.1 \\
\hline Democrats & 53.4 & 53.2 & 51.8 & 48.2 & 53.4 & 51.7 & $\underline{53.5}$ & 51.8 \\
\hline \multicolumn{9}{|l|}{ Error of national predictions } \\
\hline Average absolute error for all parties & & 2.7 & 3.2 & 4.8 & 2.5 & 3.0 & $\underline{2.4}$ & 2.9 \\
\hline Average absolute error of D-R margin & & 3.7 & 1.6 & -3.1 & 3.8 & 1.2 & 3.7 & $\underline{1.1}$ \\
\hline \multicolumn{9}{|l|}{ Average error of state predictions } \\
\hline Average absolute error for all parties & & 6.6 & 5.7 & 5.8 & 6.3 & 4.9 & 6.2 & $\underline{4.8}$ \\
\hline
\end{tabular}

Note. BTS = Bayesian Truth Serum. BTSD = Bayesian Truth Serum with divergence score (see Eq. 1). Bold underline indicates predictions with lowest error within the USC survey. National predictions are average of state predictions weighted by the size of a state population $18+$ 


\section{Figures for additional analyses}
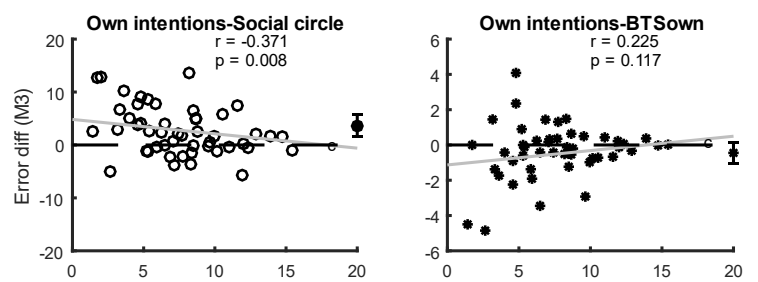

All parties: Difference in error (M3)
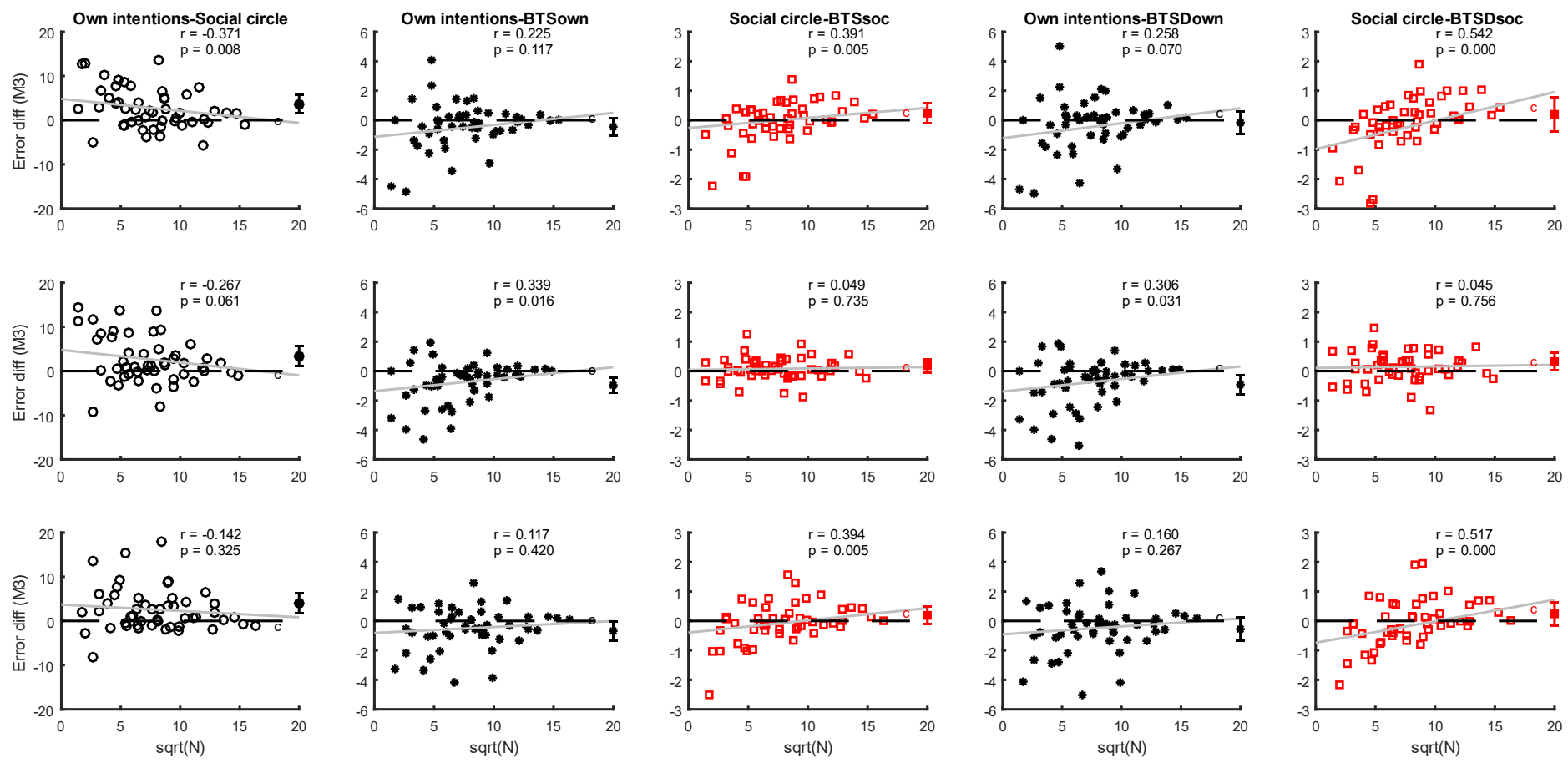

Figure S1. Differences in errors produced by different prediction methods for vote shares of all parties in each state (Mosteller 3), as a function of the square root of the survey sample size in each state. All error differences are calculated against the error of own intentions. If the difference is below 0 , then own intentions perform better. If the difference is above 0 , then the alternative method performs better. Top row: Wave 1, Middle row: Wave 2, and Bottom row: Wave 3. Each panel shows differences in errors of two prediction methods for individual states (empty markers), averaged across states and weighted by the square root of the survey sample size (full markers at the right-hand side of each panel), with 95\% confidence intervals, and Spearman correlations between the square root of sample size and the difference between errors $(r)$ with corresponding $p$ values. California is shown as a $c$ before the average difference, because of its very large sample size ( $n=1146-1305)$. 
D-R margin: Difference in error (M5)
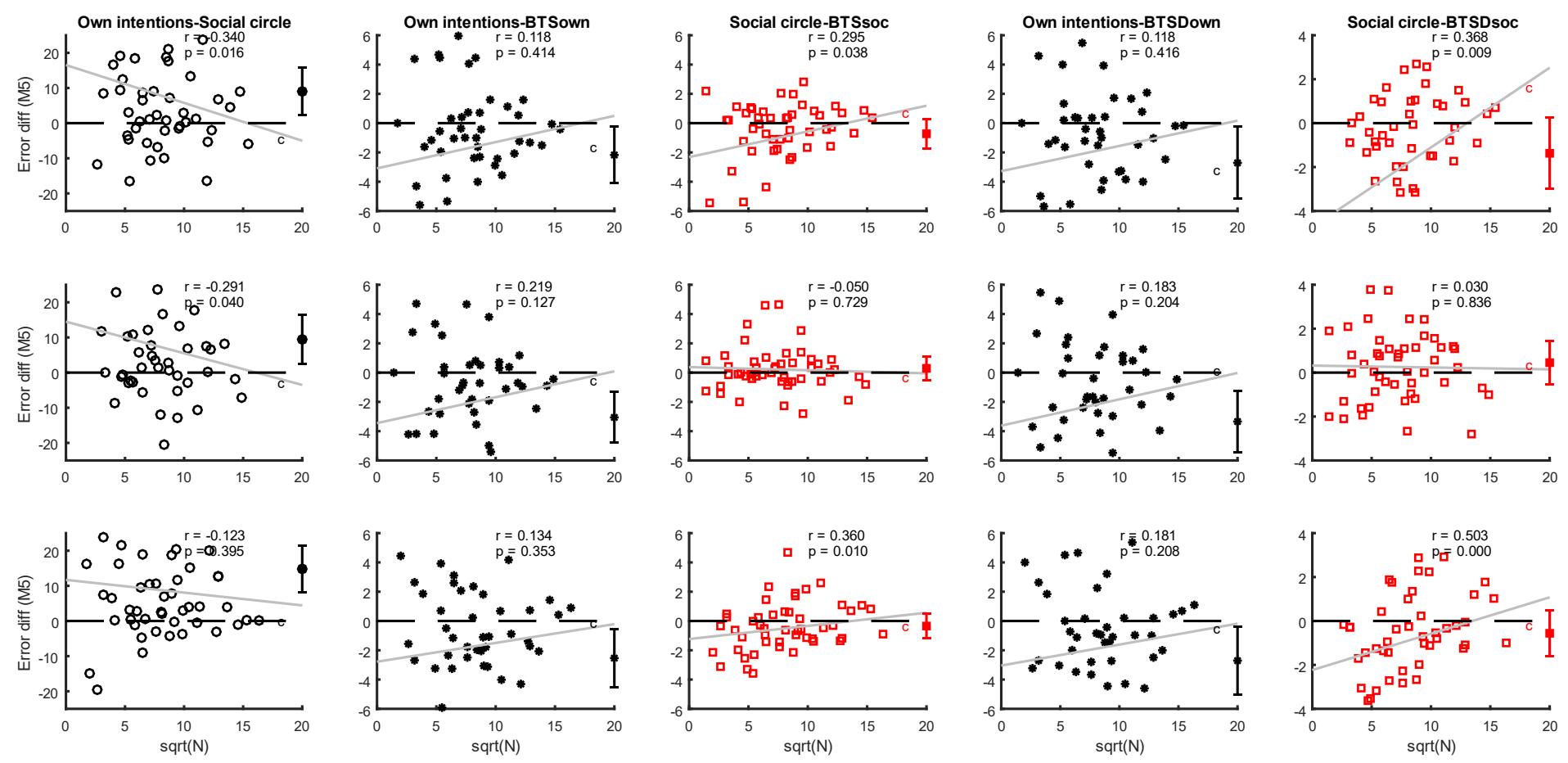

Figure S2. Differences in errors produced by different prediction methods for the D-R margin in each state (Mosteller 5) as a function of the square root of the survey sample size in each state. If the difference is below 0 , then own intentions perform better. If the difference is above 0 , then the alternative method perform better. Top row: Wave 1, Middle row: Wave 2, and Bottom row: Wave 3. Each panel shows differences in errors of two prediction methods for individual states (empty markers), averaged across states and weighted by the square root of the survey sample size (full markers at the right-hand side of each panel) . with 95\% confidence intervals, and Spearman correlations between the square root of the sample size and the difference between errors $(r)$ with corresponding $p$ values. California is shown as a $c$ before the average difference, because of its very large sample size $(n=1146-1305)$. 
Error for all parties (M3)
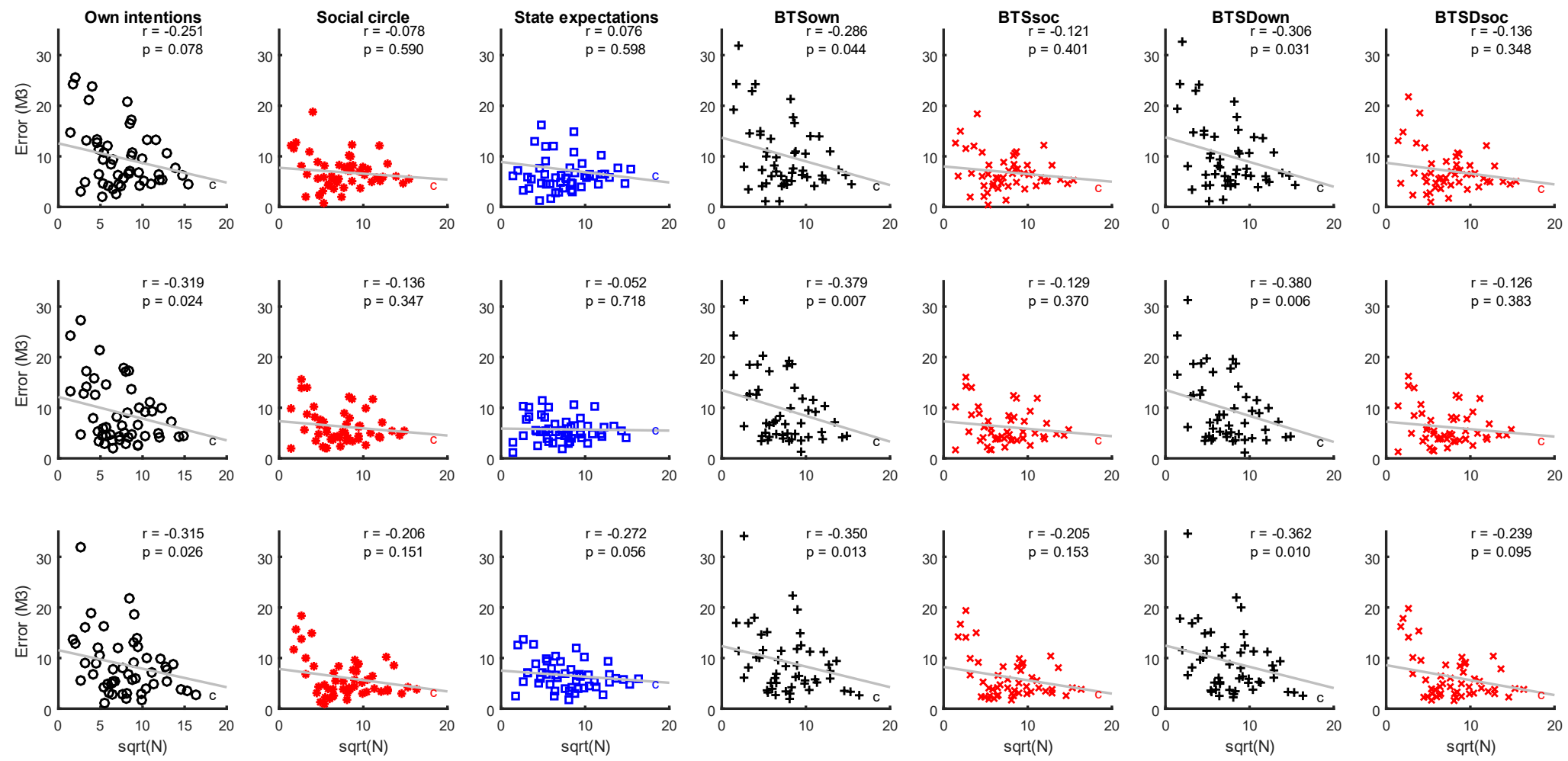

Figure S3. Error of predicting vote shares for all parties (Mosteller 3) for different square root of survey sample sizes $(N)$ in each state, and correlations $(r)$ with corresponding $p$ values between state error and the survey sample size in each state. California is shown as a $c$ and moved down to $\operatorname{sqrt}(N)=18$, because of its very large sample size $(N=1146-1305)$. 
D-R margin (M5)
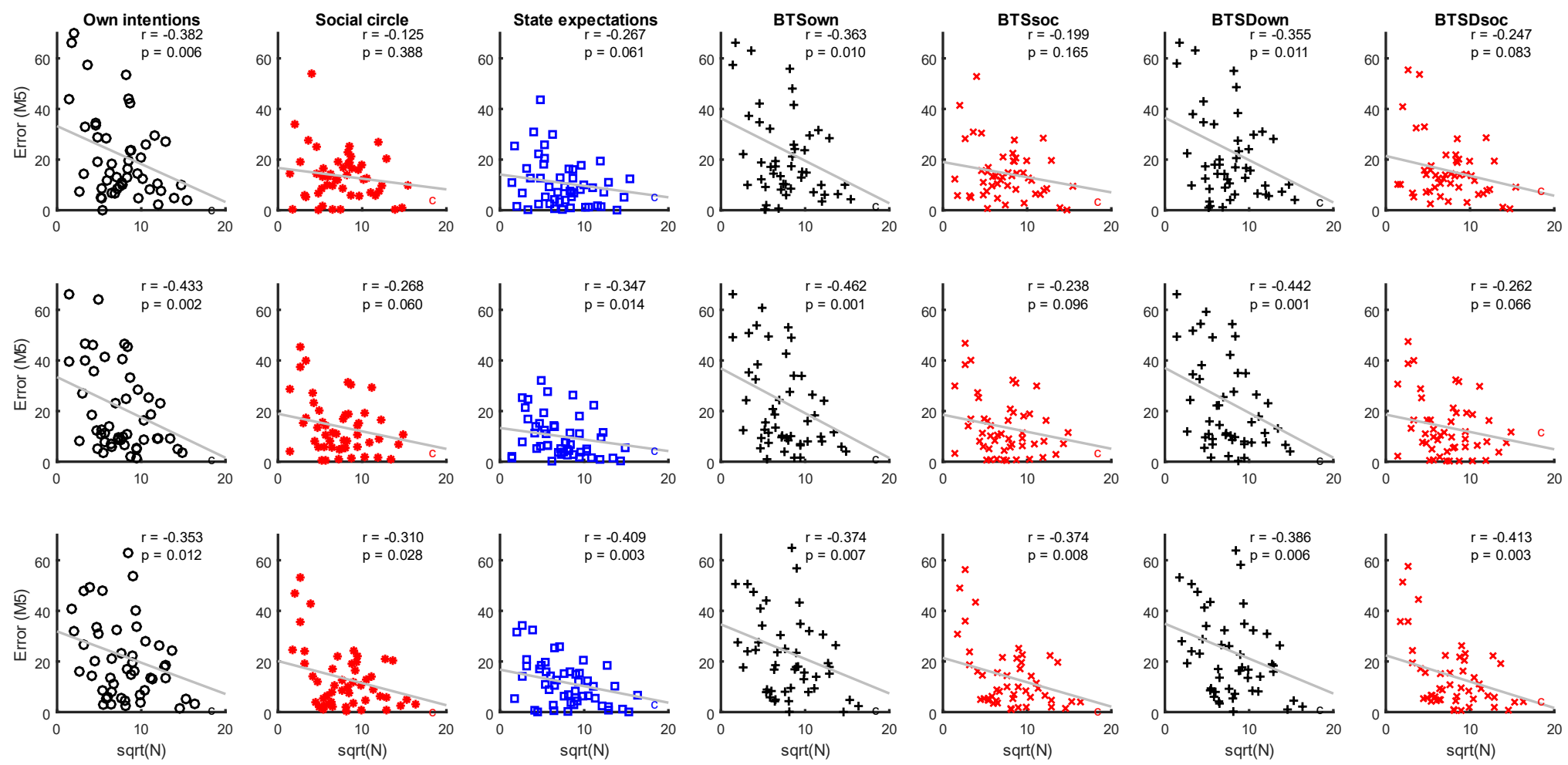

Figure S4. Errors of predicting the D-R margin (Mosteller 5) for different square root of survey sample sizes $(N)$ in each state, and correlations $(r)$ with corresponding $p$ values between state error and the survey sample size in each state. California is shown as a $\mathrm{c}$ and moved down to $\operatorname{sqrt}(N)=18$, because of its very large sample size $(N=1146-1305)$. 


\section{Simulation results}
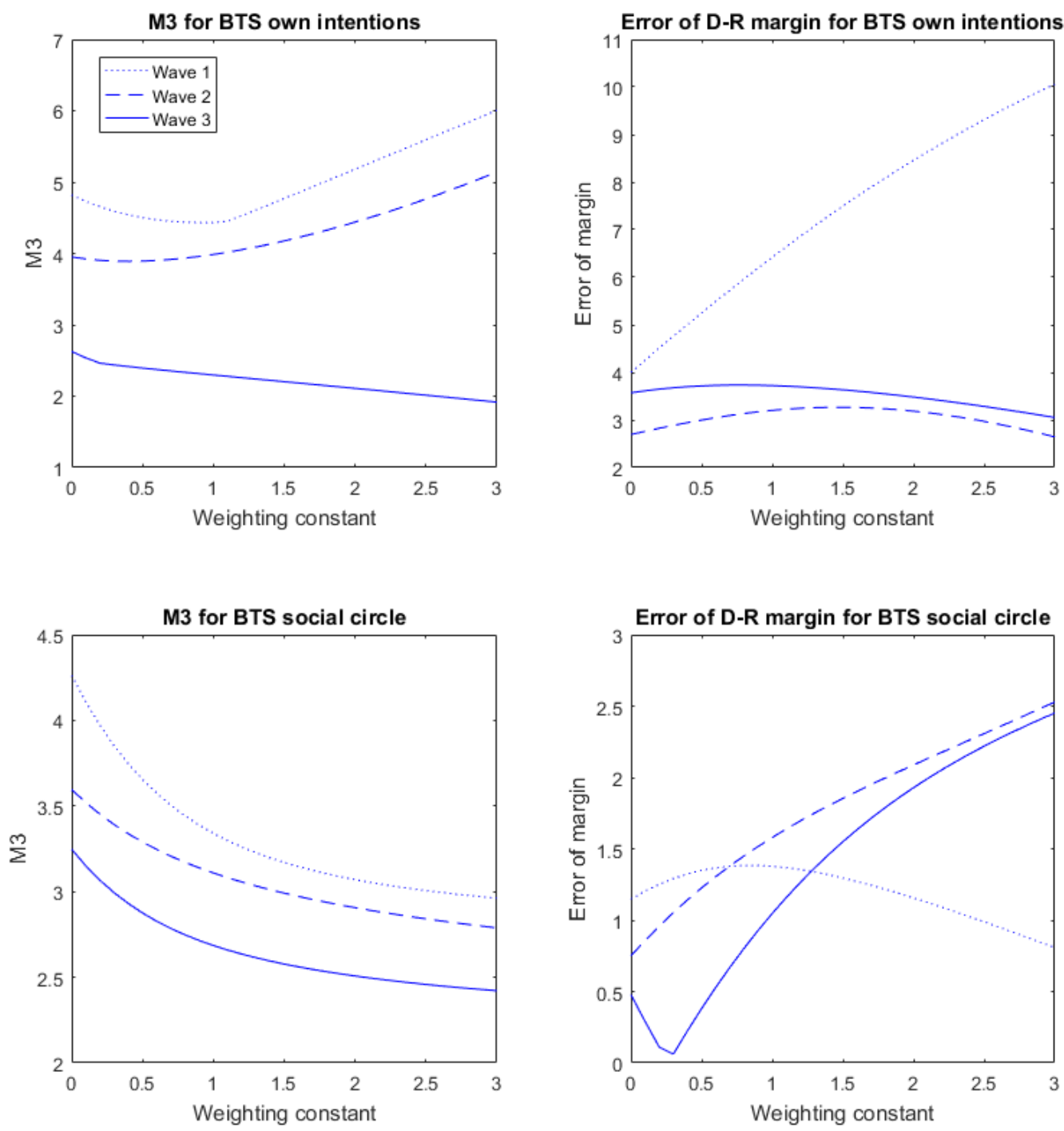

Figure S5. Exploring the effect of the weighting constant on BTS scores. Average absolute error of predictions of vote shares (Mosteller 3) and absolute error of predictions of the D-R margin (Mosteller 5) for different values of the weighting constant, for each of the three waves. 

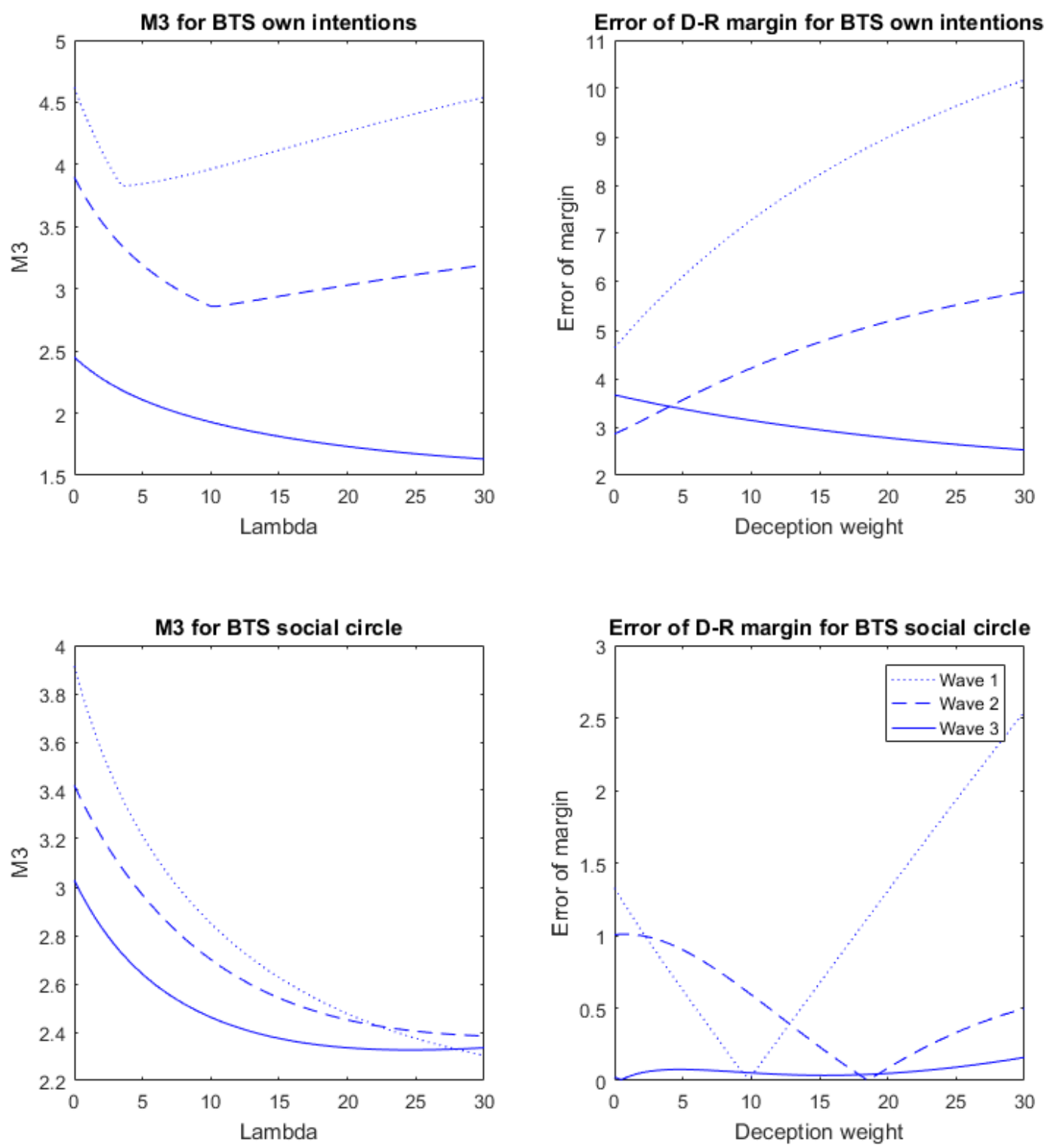

Figure S6. Exploring the effect of the weighting constant on BTSD scores. Average absolute error of predictions of vote shares (Mosteller 3) and absolute error of predictions of the D-R margin (Mosteller 5) for different values of lambda, for each of the three waves (weighting constant fixed to 0.25). 


\section{Preregistered hypotheses}

We preregistered the following hypotheses (see (39) for a full description):

H1a. Based on previous research that found that social-circle expectations outperformed own intentions in election predictions (Galesic et al., 2018), we hypothesize that predictions based on social-circle expectations will be more accurate than predictions based on own intentions.

H1b. Based on previous research that found that winner expectations outperformed own intentions in election predictions (Graefe, 2014), we hypothesize that predictions based on statewinner expectations will be more accurate than predictions based on own intentions.

H2a. Based on the theoretical properties of the Bayesian Truth Serum (BTS, Prelec, 2004), we hypothesize that applying a variant of the Bayesian Truth Serum will increase the accuracy of predictions based on own intentions.

H2b. Based on the theoretical properties of the Bayesian Truth Serum (BTS, Prelec, 2004), we hypothesize that applying a variant of the Bayesian Truth Serum will increase the accuracy of predictions based on social-circle expectations.

H3. Based on previous research that found that social-circle expectations predicted actual voting behavior over and above own intentions and winner expectations (Galesic et al., 2018), we hypothesize that social-circle expectations will predict actual individual voting behavior over and above own intentions and state-winner expectations.

We expected that hypotheses 1 and 2 will hold for predictions on three levels: 1) national vote shares obtained from the whole survey sample, 2) national vote shares obtained from the average of state level predictions, 3) state vote shares obtained from the survey sample in each state. The exceptions are the predictions based on BTS, as they can only be applied to level 2 and 3. Hypothesis 3 is applied to the individual level and focuses on predicting votes for either a Democratic or Republican candidate.

Predictions based on social-circle expectations will be more accurate than predictions based on own intentions (H1a). Social-circle expectations outperformed own intentions in predicting election results for the majority of the comparisons on the national and state levels (Tables 1, S1, Figures 1 and 2), in line with H1a (Figures 1 and 2). The only exception was in the third wave in predicting the election results for all parties on the national 
level (Figure 1A). Out of 50 states, social circle-expectations were more accurate than own intentions for 32 to 38 states (depending on the survey wave and error measure).

State-winner expectations will be more accurate than predictions based on own intentions (H1b). State-winner expectations were mostly worse than own intentions for predictions on the national level (Figure 1, Table S1), contrary to H1b. The one exception was for Wave 1 for the D-R margin where it outperformed own intentions (Figure 1B).

However, in line with H1b, state-winner expectations consistently outperformed own intentions on the state level both for all parties and the D-R margin (Figure 2). In fact, statewinner expectations outperformed all other methods for the D-R margin on the state level (Figure 2B). Out of 50 states, state-winner expectations were more accurate than own intentions for 30 to 36 states, depending on survey wave and error measure.

\section{Bayesian Truth Serum will increase the accuracy of predictions based on own} intentions (H2a). See Results section.

\section{Bayesian Truth Serum will increase the accuracy of predictions based on social-} circle expectations (H2b). See Results section.

\section{Social-circle expectations will predict actual individual voting behavior over and} above own intentions and state-winner expectations (H3). Social-circle expectations predict individual voting for a Republican or a Democrat candidate over and above own intentions, in line with H3. This is suggested by the results from a multiple regression model that included own intentions and social-circle expectations (Table S5 to S7). Own intentions were the best predictors across the three groups of participants, with $b$ values close to 1 for the participants that voted either Republican or Democratic. The $b$ values for social circle expectations were between 0.06 and on 0.08 . Contrary to $\mathrm{H} 3 \mathrm{a}$, social-circle expectations did not reliably predict voting behavior for those who voted for Other candidates. This last result should be interpreted with care as, unlike for those who voted Republican or Democrat (adjusted R-square $=0.77$ and 0.78), the model for Other does not fit those data very well (adjusted R-square $=0.1$ ).

State-winner expectations did not predict additional variance in individual voting behavior for most participants, in line with $\mathrm{H} 3 \mathrm{~b}$. However, they did help improve the predictions of voting behavior of those participants who voted for Other candidates. As with the analysis for the simpler model with only own intentions and social-circle expectations, this result should be interpreted with care as the model for Other does not fit data very well (adjusted R-square $=0.1$ ). 


\section{Predicting individual voting behavior}

Table S5

Results of linear regressions predicting reported voting behavior from own intentions, social circle expectations, and state expectations reported in wave 3

\begin{tabular}{|c|c|c|c|}
\hline & \multicolumn{3}{|c|}{ Own voting behavior reported post-election } \\
\hline & $\begin{array}{l}\text { Voted Republican } b \\
\text { (SE) }\end{array}$ & $\begin{array}{l}\text { Voted Democratic } b \\
\text { (SE) }\end{array}$ & Voted Other $b(\mathrm{SE})$ \\
\hline \multicolumn{4}{|c|}{ Model with only own intentions } \\
\hline \multirow[t]{2}{*}{ Own intentions } & $0.99 * * *$ & $0.99 * * *$ & $0.37 * * *$ \\
\hline & $(0.01)$ & $(0.01)$ & $(0.02)$ \\
\hline \multirow[t]{2}{*}{ Constant } & 0.73 & $2.56 * * *$ & $0.92 * * *$ \\
\hline & $(0.63)$ & $(0.67)$ & $(0.31)$ \\
\hline Adj. $R$-square & 0.78 & 0.78 & 0.10 \\
\hline \multicolumn{4}{|c|}{ Model with own intentions and social-circle expectations } \\
\hline \multirow[t]{2}{*}{ Own intentions } & $0.95 * * *$ & $0.97 * * *$ & $0.36 * * *$ \\
\hline & $(0.01)$ & $(0.01)$ & $(0.03)$ \\
\hline Social-circle & $0.079 * * *$ & $0.059 * *$ & 0.03 \\
\hline expectations & $(0.02)$ & $(0.02)$ & $(0.04)$ \\
\hline \multirow[t]{2}{*}{ Constant } & -1.29 & 0.99 & $0.82 *$ \\
\hline & $(0.83)$ & $(0.88)$ & $(0.33)$ \\
\hline Adj. $R$-square & 0.77 & 0.78 & 0.10 \\
\hline \multicolumn{4}{|c|}{ Model with own intentions, social-circle expectations, and state expectations } \\
\hline \multirow[t]{2}{*}{ Own intentions } & $0.95 * * *$ & $0.96 * * *$ & $0.35 * * *$ \\
\hline & $(0.01)$ & $(0.01)$ & \\
\hline Social-circle & $0.07 * * *$ & $0.05^{*}$ & -0.03 \\
\hline expectations & $(0.02)$ & $(0.02)$ & $(0.05)$ \\
\hline State-winner & 0.01 & 0.02 & $0.10^{*}$ \\
\hline expectations & $\left(0.03 \_\right.$ & $(0.03)$ & $(0.05)$ \\
\hline \multirow[t]{2}{*}{ Constant } & -1.61 & 0.48 & 0.60 \\
\hline & (1.09) & (1.16) & $(0.35)$ \\
\hline Adj. $R$-square & 0.77 & 0.77 & 0.10 \\
\hline
\end{tabular}

Note. $N=2,859$. 
Table S6

Results of linear regressions predicting reported voting behavior from own intentions, social circle expectations, and state expectations reported in wave 2

\begin{tabular}{|c|c|c|c|}
\hline & \multicolumn{3}{|c|}{ Own voting behavior reported post-election } \\
\hline & $\begin{array}{l}\text { Voted Republican } b \\
\text { (SE) }\end{array}$ & $\begin{array}{l}\text { Voted Democratic } b \\
(\mathrm{SE})\end{array}$ & Voted Other $b(\mathrm{SE})$ \\
\hline \multicolumn{4}{|c|}{ Model with only own intentions } \\
\hline \multirow[t]{2}{*}{ Own intentions } & $0.99 * * *$ & $0.99 * * *$ & $0.26 * * *$ \\
\hline & $(0.01)$ & $(0.01)$ & $(0.02)$ \\
\hline \multirow[t]{2}{*}{ Constant } & $1.51 *$ & $2.94 * * *$ & $1.15 * * *$ \\
\hline & $(0.68)$ & $(0.75)$ & $(0.32)$ \\
\hline Adj. $R$-square & 0.74 & 0.73 & 0.06 \\
\hline \multicolumn{4}{|c|}{ Model with own intentions and social-circle expectations } \\
\hline \multirow[t]{2}{*}{ Own intentions } & $0.95 * * *$ & $0.95 * * *$ & $0.25 * * *$ \\
\hline & $(0.02)$ & $(0.02)$ & $(0.02)$ \\
\hline Social-circle & $0.08 * * *$ & $0.08 * * *$ & 0.03 \\
\hline expectations & $(0.02)$ & $(0.02)$ & $(0.03)$ \\
\hline \multirow{2}{*}{ Constant } & -0.67 & 0.82 & $1.05 * *$ \\
\hline & $(0.90)$ & $(0.98)$ & $(0.35)$ \\
\hline Adj. $R$-square & 0.74 & 0.73 & 0.06 \\
\hline \multicolumn{4}{|c|}{ Model with own intentions, social-circle expectations, and state expectations } \\
\hline \multirow[t]{2}{*}{ Own intentions } & $0.955 * * *$ & $0.95 * * *$ & $0.24 * * *$ \\
\hline & $(0.0151)$ & $(0.02)$ & $(0.02)$ \\
\hline Social-circle & $0.11 * * *$ & $0.09 * * *$ & -0.04 \\
\hline expectations & $(0.03)$ & $(0.03)$ & $(0.04)$ \\
\hline \multirow[t]{2}{*}{ State expectations } & -0.05 & -0.03 & $0.12 * *$ \\
\hline & $(0.03)$ & $(0.03)$ & $(0.04)$ \\
\hline \multirow[t]{2}{*}{ Constant } & 0.65 & 1.63 & $0.76^{*}$ \\
\hline & $(1.15)$ & $(1.29)$ & $(0.36)$ \\
\hline Adj. $R$-square & 0.74 & 0.73 & 0.06 \\
\hline
\end{tabular}

Note. $N=2,859$. 
Table S7

Results of linear regressions predicting reported voting behavior from own intentions, social circle expectations, and state expectations reported in wave 1

\begin{tabular}{|c|c|c|c|}
\hline & \multicolumn{3}{|c|}{ Own voting behavior reported post-election } \\
\hline & $\begin{array}{l}\text { Voted Republican } b \\
\text { (SE) }\end{array}$ & $\begin{array}{l}\text { Voted Democratic } b \\
\text { (SE) }\end{array}$ & Voted Other $b(\mathrm{SE})$ \\
\hline \multicolumn{4}{|c|}{ Model with only own intentions } \\
\hline \multirow[t]{2}{*}{ Own intentions } & $0.99 * * *$ & $0.979 * * *$ & $0.22 * * *$ \\
\hline & $(0.01)$ & $(0.01)$ & $(0.01)$ \\
\hline \multirow[t]{2}{*}{ Constant } & $1.97 * *$ & $4.03 * * *$ & $1.13 * * *$ \\
\hline & $(0.70)$ & $(0.79)$ & $(0.33)$ \\
\hline Adj. $R$-square & 0.72 & 0.70 & 0.05 \\
\hline \multicolumn{4}{|c|}{ Model with own intentions and social-circle expectations } \\
\hline \multirow[t]{2}{*}{ Own intentions } & $0.94 * * *$ & $0.92 * * *$ & $0.17 * * *$ \\
\hline & $(0.02)$ & $(0.02)$ & $(0.02)$ \\
\hline Social-circle & $0.11 * * *$ & $0.15 * * *$ & $0.08 *$ \\
\hline expectations & $(0.02)$ & $(0.02)$ & $(0.04)$ \\
\hline \multirow{2}{*}{ Constant } & -0.81 & 0.2 & $0.08 *$ \\
\hline & $(0.92)$ & (1) & $(0.04)$ \\
\hline Adj. $R$-square & 0.72 & 0.70 & 0.06 \\
\hline \multicolumn{4}{|c|}{ Model with own intentions, social-circle expectations, and state expectations } \\
\hline \multirow[t]{2}{*}{ Own intentions } & $0.94 * * *$ & $0.92 * * *$ & $0.17 * * *$ \\
\hline & $(0.02)$ & $(0.02)$ & $(0.02)$ \\
\hline Social-circle & $0.13 * * *$ & $0.15 * * *$ & $0.08^{*}$ \\
\hline expectations & $(0.03)$ & $(0.03)$ & $(0.04)$ \\
\hline \multirow[t]{2}{*}{ State expectations } & -0.05 & -0.02 & $0.08 *$ \\
\hline & $(0.03)$ & $(0.03)$ & $(0.04)$ \\
\hline \multirow[t]{2}{*}{ Constant } & 0.56 & 0.66 & 0.50 \\
\hline & (1.19) & $(1.33)$ & $(0.36)$ \\
\hline Adj. $R$-square & 0.72 & 0.70 & 0.06 \\
\hline
\end{tabular}

Note. $N=2,859$. 